Anke Petschenka, Richard Stang, Alexandra Becker, Fabian Franke, Christine Gläser, Hans-Dieter Weckmann und Bert Zulauf

\title{
Die Zukunft der Lernwelt Hochschule gestalten
}

\author{
Ein Baukasten für Veränderungsprozesse
}

\section{Einleitung}

Die Gestaltung von Lernwelten an Hochschulen stellt eine multidimensionale Herausforderung dar. Auf der einen Seite ist sie ein zentraler Auftrag der Hochschule als Bildungsinstitution, auf der anderen Seite spielen die unterschiedlichsten Akteurinnen und Akteure eine bedeutende Rolle dabei. Ausgehend von den politischen Rahmenbedingungen gestalten Hochschulleitung, Hochschulverwaltung, IT- und Infrastrukturabteilungen, Bibliotheken und Medienzentren sowie ganz grundsätzlich die Lehrenden und Studierenden die Lernwelt einer Hochschule. Doch ist dies keine triviale Aufgabe.

Im Projekt Lernwelt Hochschule (LeHo) wurden die Handlungsstrukturen der einzelnen Akteurinnen und Akteure untersucht, sowie Good Practice-Beispiele genauer in den Blick genommen. Auf Basis der Analyse wurden zentrale Herausforderungen sichtbar (Stang et al. 2020). Im Projekt ging es auch darum, herauszufinden, welche Faktoren bei der Bewältigung dieser Herausforderungen eine besondere Rolle spielen und welche Stellschrauben gedreht werden können, um die jeweilige Lernwelt Hochschule zu gestalten. Aus den Forschungsergebnissen wurde ein Baukasten (Handlungskontext) gegossen, mit dem Anregungen zur Gestaltung der Lernwelt Hochschule, unter anderem auch von Gebäuden mit Lernflächen oder Lernräumen, für verantwortliche Akteurinnen und Akteure bereitgestellt werden sollen. Der Baukasten dient sowohl als Checkliste als auch als Anregung, über den Tellerrand zu blicken und umfassende zeitgemäße Konzepte zu realisieren. Dabei soll insbesondere die studentische Perspektive in allen Planungsphasen berücksichtigt werden.

Als theoretische Grundlage für weiterführende Überlegungen dient das PST Framework for Designing and Evaluation Learning Places (Radcliffe et al. 2009). Die von Radcliffe et al. angeführten Komponenten „Space - Technology Pedagogy“ sollen um weitere Komponenten erweitert beziehungsweise differenzierter betrachtet werden: 
1. Space: Bau und Lernraumgestaltung,

2. Technology: Digitale Struktur,

3. Pedagogy: Didaktik.

Nach Auswertung der Forschungsergebnisse des Projekts Lernwelt Hochschule wird als Erweiterung des PST Frameworks die Komponente Hochschulorganisation ergänzt.

Der neue Handlungsrahmen sieht folgende Struktur vor (Abbildung 1):

1. Hochschulorganisation,

2. Hochschuldidaktik,

3. Digitale Strukturen,

4. Physische Lehr- und Lernräume.

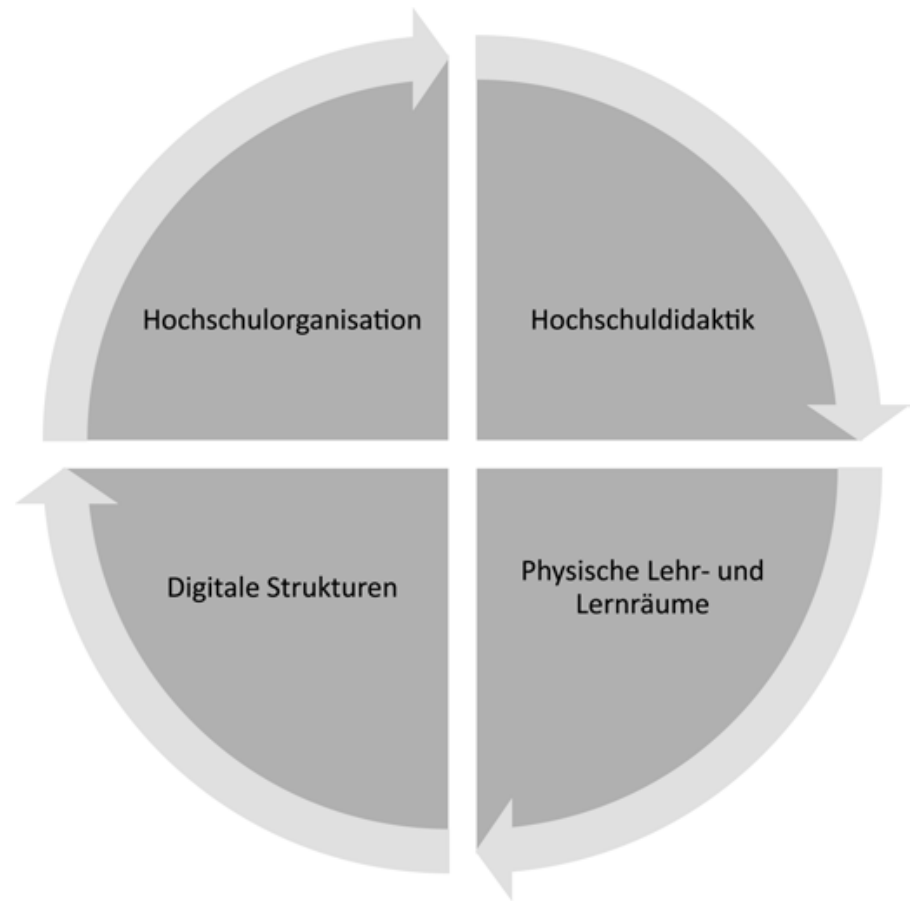

Abb. 1: Die vier Themenfelder des Baukastens Lernwelt Hochschule.

Gerahmt werden sie durch die übergeordnete Dachkonstruktion Hochschulpolitik. Ohne diesen entscheidenden Faktor sind alle weitergehenden Überlegungen auf operativer Ebene nur schwer umzusetzen. Die politischen Entscheidungsträ- 
gerinnen auf Landesebene - für Hochschule zuständige Ministerien - sind zugleich auch für die Finanzierung von landesweiten beziehungsweise lokalen Hochschulaktivitäten zuständig. Deshalb spielt auch diese Ebene im Baukasten eine Rolle. Im Fokus stehen allerdings die vier Bereiche Hochschulorganisation, Hochschuldidaktik, digitale Strukturen und physische Lehr- und Lernräume. Dabei macht die Betrachtung dieser vier Bereiche deutlich,

- dass eine Verzahnung der vier Bereiche im Sinne einer ganzheitlichen Betrachtung nicht nur sinnvoll, sondern notwendig ist,

- dass jeder Bereich seine eigenen Logiken hat, die unter der Perspektive der jeweiligen Akteurinnen und Akteure und bezogen auf eine übergreifende Hochschulstrategie zu betrachten sind.

Die Herausforderungen, mit denen Hochschulen bei der Konzeption, Planung und Umsetzung von Lernwelten konfrontiert sind (Stang et al. 2020), werden in einen Baukasten eingegliedert und adressieren verschiedene Akteurinnen und Akteure an Hochschulen:

- Hochschulleitung,

- Organisationseinheiten der Hochschule (z. B. IT- und Infrastrukturabteilungen, Didaktikzentren, Bibliotheken und Medienzentren),

- Hochschullehrende,

- Studierende.

Die hier angeschnittenen Themen des Baukastens finden eine thematische Vertiefung im Wiki des Projektes Lernwelt Hochschule ${ }^{1}$.

Im Folgenden soll eine Strukturierung der Herausforderungen in den vier Bereichen vorgenommen und im Überblick mögliche Perspektiven durch Handlungsempfehlungen eröffnet werden. Auch für das übergeordnete Themenfeld Hochschulpolitik sollen Handlungsempfehlungen formuliert werden. Den Autorinnen und Autoren ist bewusst, dass viele der aufgeführten Herausforderungen von Hochschulen bereits bearbeitet werden - auch dies ist ein Ergebnis des Projektes Lernwelt Hochschule. Doch handelt es sich oft um Einzelaktivitäten, die nicht selten auf der Basis von Projektförderung realisiert werden. Im Rahmen des Baukastens geht es nun darum, die verschiedenen Perspektiven zu bündeln, um die Hochschule als Lernwelt ganzheitlich zu betrachten.

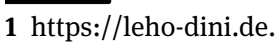




\section{Auf einen Blick}

\section{Hochschulpolitik}

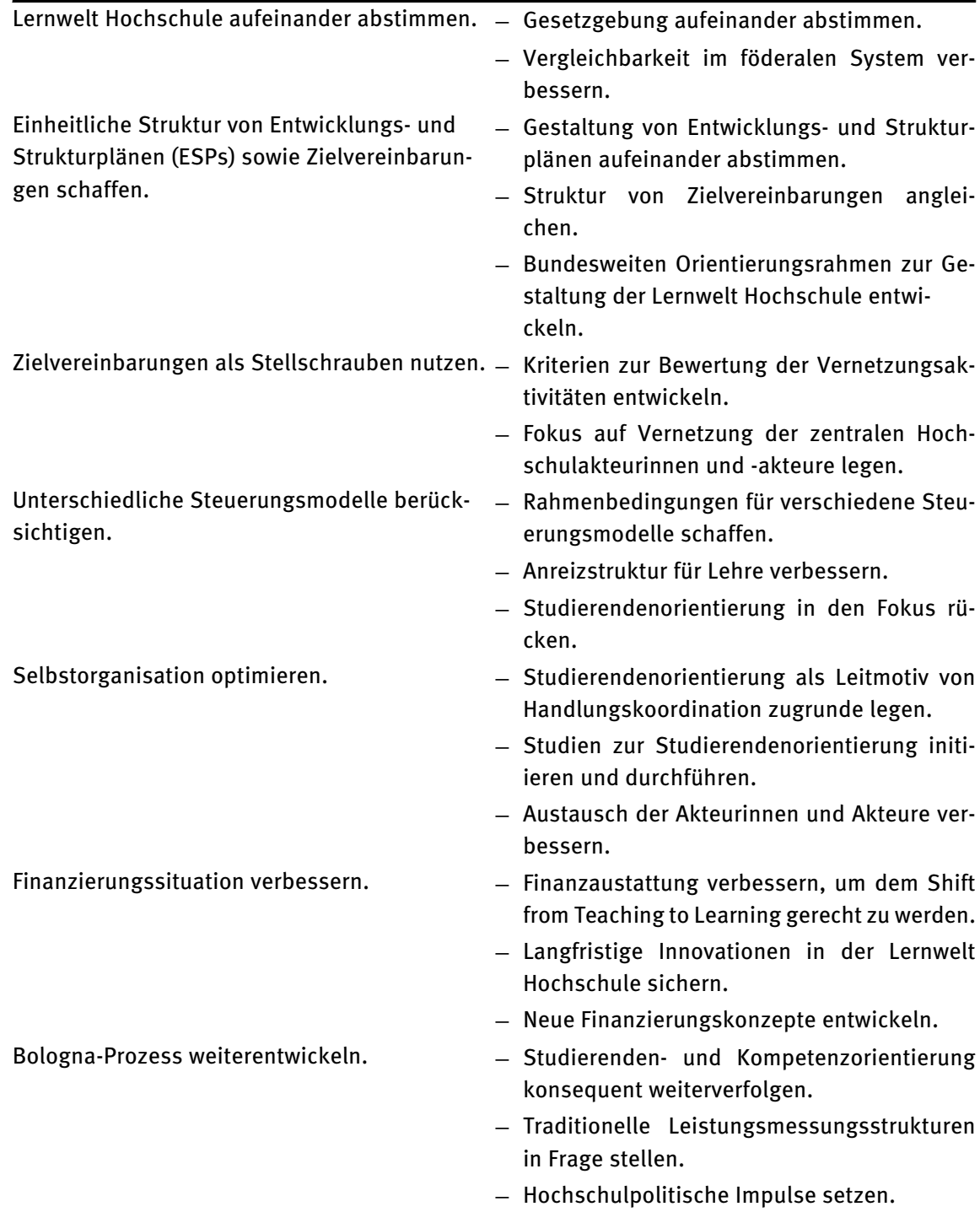

- Struktur von Zielvereinbarungen angleichen.

- Bundesweiten Orientierungsrahmen zur Gestaltung der Lernwelt Hochschule entwickeln.

Zielvereinbarungen als Stellschrauben nutzen. - Kriterien zur Bewertung der Vernetzungsaktivitäten entwickeln.

- Fokus auf Vernetzung der zentralen Hochschulakteurinnen und -akteure legen.

Unterschiedliche Steuerungsmodelle berück- - Rahmenbedingungen für verschiedene Steusichtigen. erungsmodelle schaffen.

- Anreizstruktur für Lehre verbessern.

- Studierendenorientierung in den Fokus rücken.

Selbstorganisation optimieren.

- Studierendenorientierung als Leitmotiv von Handlungskoordination zugrunde legen.

- Studien zur Studierendenorientierung initiieren und durchführen.

- Austausch der Akteurinnen und Akteure verbessern.

Finanzierungssituation verbessern.

- Finanzaustattung verbessern, um dem Shift from Teaching to Learning gerecht zu werden.

- Langfristige Innovationen in der Lernwelt Hochschule sichern.

- Neue Finanzierungskonzepte entwickeln.

Bologna-Prozess weiterentwickeln.

- Studierenden- und Kompetenzorientierung konsequent weiterverfolgen.

- Traditionelle Leistungsmessungsstrukturen in Frage stellen.

- Hochschulpolitische Impulse setzen. 


\begin{tabular}{|c|c|}
\hline Reformprozesse fördern. & $\begin{array}{l}\text { - Ganzheitliche Innovationskorridore schaf- } \\
\text { fen. } \\
\text { - Konzertierte Aktivitäten von Hochschulpoli- } \\
\text { tik und Hochschulen initiieren. }\end{array}$ \\
\hline Transparente Datenstruktur erzeugen. & $\begin{array}{l}\text { - Datenbasis zur Studierendenorientierung } \\
\text { schaffen. } \\
\text { - Entwicklungs- und Strukturpläne sowie Ziel- } \\
\text { vereinbarungen bundesweit dokumentieren. } \\
\text { - Lern-Management-Systeme und physische } \\
\text { Lehr- und Lernräume kartieren. } \\
\text { - Bundesweit agierende Forschungsinstituti- } \\
\text { on etablieren. }\end{array}$ \\
\hline Entwicklung dynamisieren. & $\begin{array}{l}\text { - Hochschulpolitischer Entscheidungsprozes- } \\
\text { se effektivieren und beschleunigen. } \\
\text { - Traditionelle Hochschulkulturen aufbrechen. } \\
\text { - Verändertes Innovationsklima schaffen. } \\
\text { - Bürokratische Strukturen reduzieren. }\end{array}$ \\
\hline
\end{tabular}

Aus der Perspektive der jeweiligen Hochschulakteurinnen und -akteure ergeben sich auf der Ebene der Hochschulpolitik kaum Handlungsoptionen. Gremien wie die Hochschulrektorenkonferenz könnten hier Veränderungen einfordern. Die letztendliche Entscheidung über gesetzliche Veränderungen treffen die Landesparlamente. Einzelne Regelungen können von den jeweiligen Landesministerien spezifiziert werden. Auch gibt es die Möglichkeit, mit Bund-Länder-Programmen die Rahmenbedingungen für die Arbeit der Hochschulen zu gestalten.

\section{Hochschulorganisation}

\begin{tabular}{ll}
\hline Gesamtstrategie in den Blick nehmen. & - Ganzheitliche Perspektive einnehmen. \\
- & Beteiligung aller Hochschulakteurinnen und \\
& -akteure sicherstellen. \\
- & Interne Kooperationsmodelle entwickeln. \\
& - Gratifikationssystem für Lehrexzellenz ent- \\
Ungleichgewicht von Forschung und Lehre be- & wickeln. \\
seitigen. & - Kriterienraster zur Bewertung exzellenter \\
& Lehre entwickeln.
\end{tabular}




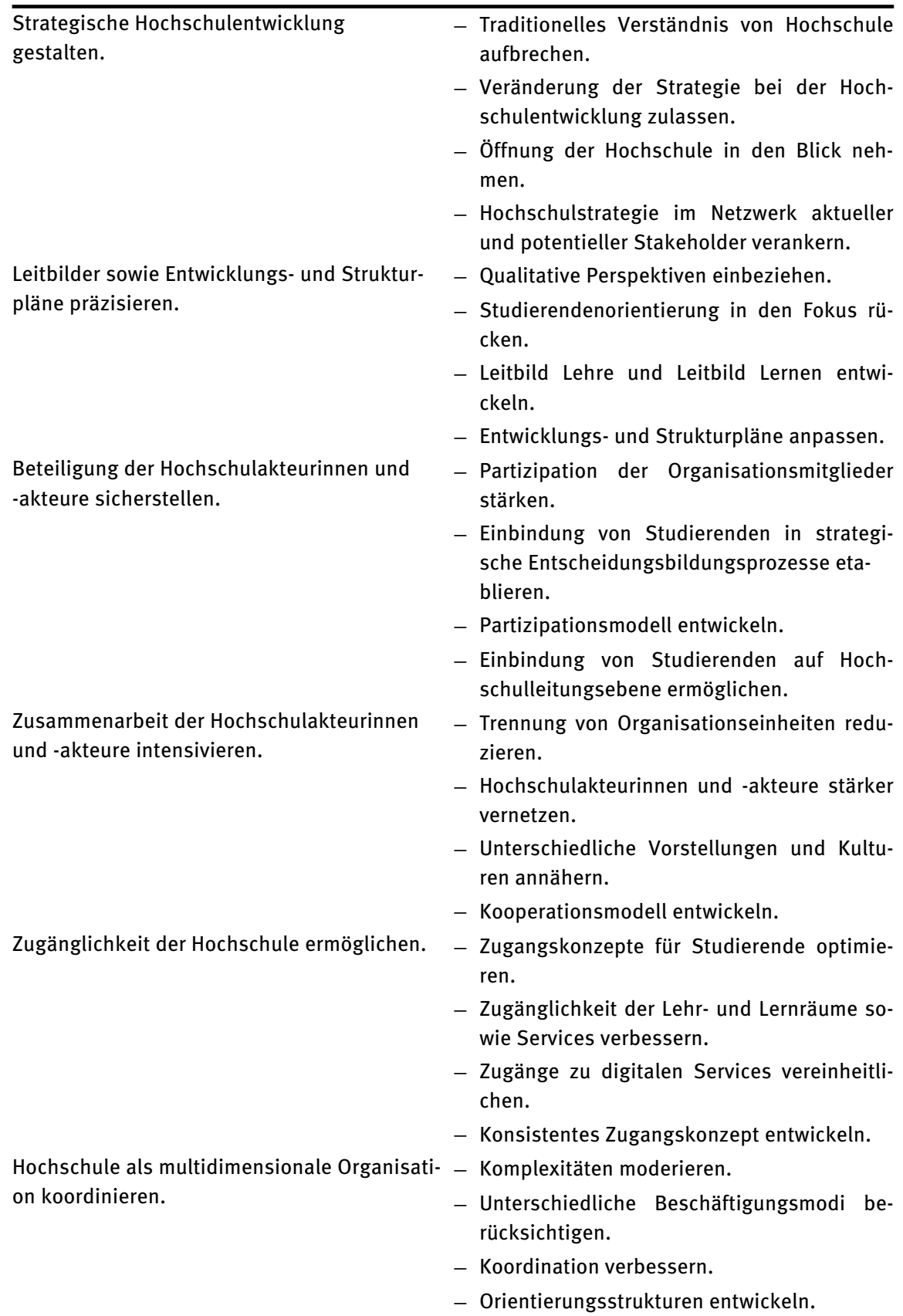




\begin{tabular}{ll}
\hline $\begin{array}{l}\text { Ganzheitliche und integrierte Lernwelt entwi- } \\
\text { ckeln. }\end{array}$ & - Hochschule ganzheitlich steuern. \\
& - Beteiligung von Studierenden intensivieren. \\
& - Wissen über die Organisation Hochschule \\
& vermitteln. \\
& - Anreizstrukturen schaffen. \\
& - Den Wandel gestalten. \\
„Atmende“ Hochschule denken. & - Agilität entwickeln. \\
& - Am Student-Life-Cycle in der Lernwelt Hoch- \\
& schule orientieren. \\
\hline
\end{tabular}

Aus der Perspektive der jeweiligen Akteurinnen und Akteure ergeben sich folgende Herausforderungen:

\section{Hochschulleitung}

- Studierendenorientierung in den Fokus rücken.

- Strategie ganzheitlich gestalten.

- Hochschulstrategie im Netzwerk aktueller und potentieller Stakeholder verankern.

- Interne Kooperationsmodelle entwickeln.

- Kriterienraster zur Bewertung exzellenter Lehre entwickeln.

- Leitbild Lehre und Leitbild Lernen entwickeln.

- Einbindung von Studierenden in strategische Entscheidungsbildungsprozesse etablieren.

- Partizipationsmodell entwickeln.

- Einbindung von Studierenden auf Hochschulleitungsebene ermöglichen.

\section{Organisationseinheiten}

- Zugänglichkeit der Lehr- und Lernräume und Services verbessern.

- Zugänge zu digitalen Services vereinheitlichen.

- Zugangskonzepte für Studierende optimieren.

- Orientierungsstrukturen entwickeln.

\section{Hochschullehrende}

- Leitbild Lehre und Leitbild Lernen entwickeln. 


\section{Studierende}

- Interesse an strategischen Entscheidungsbildungsprozessen entwickeln.

- Interesse an Wissen über die Organisation Hochschule zeigen.

\section{Hochschuldidaktik}

\begin{tabular}{ll}
\hline Shift from Teaching to Learning in den Fokus & - Veränderte didaktische Ansätze gestalten. \\
rücken. & - Kollaboratives Arbeiten ermöglichen. \\
& - Rolle der Lehrenden in Richtung einer Lern- \\
& begleitung verändern. \\
& - Veränderte Lehr-Lernszenarien gestalten. \\
& - Rahmenbedingungen schaffen. \\
Verändertes Rollenverständnis der Lehrenden & - Prozess des Lernens organisieren, initiieren \\
etablieren. & und begleiten. \\
& - Didaktischen Rahmen gestalten. \\
& - Verantwortung der Lernenden für ihr Lernen \\
& hervorheben. \\
& - Lehre als Ermöglichungsraum verstehen. \\
& - Grundlegenden Perspektivwechsel auf Sei- \\
& ten der Lehrenden einfordern. \\
& - Fortbildungsaktivitäten verstärken. \\
& - Experimentierräume für innovative Lehre \\
& etablieren. \\
& - Wissen und Kompetenzen in Kommunikati- \\
& on mit anderen generieren. \\
& - Vom Konsumieren zum Generieren anhal- \\
& ten. \\
& - Digitale, mobile Medien (BYoD) einbinden. \\
& - Methodenvielfalt gestalten. \\
& - Methodenportfolio entwickeln. \\
& - Offenheit der Lehrenden, aber auch der Stu- \\
& dierenden fördern. \\
Wissensproduktion und Kompetenzentwick- &
\end{tabular}




\begin{tabular}{ll}
\hline Didaktikzentren als Schlüsseleinrichtungen & - Vielfalt der Arbeit der Didaktikzentren er- \\
anerkennen. & kennen. \\
- & Bedeutung der Didaktikzentren hervorhe- \\
& ben. \\
- & Didaktikzentren als zentrale Schlüsselein- \\
& richtungen etablieren. \\
- & Didaktikzentren in der Hochschulorganisati- \\
& on verankern. \\
- & Schnittstellenfunktion zu den relevanten an- \\
& deren Abteilungen herstellen. \\
- & Bereich der Lehre fokussieren. \\
- & Leitbild für die Lehre entwickeln. \\
- & Bewusstsein für gute Lehrqualität entwi- \\
& ckeln. \\
Leitbild Lehre als Qualitäts- und Kultur- & Leitbild Lehre für die Außendarstellung ein- \\
merkmal positionieren. & setzen. \\
- & Akzeptanz des Lehr-Lernkonzepts von allen \\
& Lehrenden fördern. \\
\hline &
\end{tabular}

Aus der Perspektive der jeweiligen Akteurinnen und Akteure ergeben sich folgende Herausforderungen:

\section{Hochschulleitung}

- Rahmenbedingungen schaffen.

- Vielfalt der Arbeit der Didaktikzentren erkennen.

- Didaktikzentren als zentrale Schlüsseleinrichtungen etablieren.

- Bereich der Lehre fokussieren.

- Leitbild für die Lehre entwickeln.

\section{Organisationseinheiten}

- Rahmenbedingungen schaffen.

- Fortbildungsaktivitäten verstärken.

- Experimentierräume für innovative Lehre etablieren. 


\section{Hochschullehrende}

- Veränderte didaktische Ansätze gestalten.

- Kollaboratives Arbeiten ermöglichen.

- Rolle als Lehrende in Richtung einer Lernbegleitung verändern.

- Innovative Lehr-Lernszenarien gestalten.

- Prozess des Lernens organisieren, initiieren und begleiten.

- Didaktischen Rahmen gestalten.

- Lehre als Ermöglichungsraum verstehen.

- Grundlegenden Perspektivwechsel vornehmen.

- Digitale, mobile Medien einbinden.

- Methodenvielfalt gestalten.

- Methodenportfolio entwickeln.

- Bewusstsein für gute Lehrqualität entwickeln.

- Akzeptanz des Lehr-Lernkonzepts von allen Lehrenden fördern.

\section{Studierende}

- Konsumentenhaltung aufgeben.

- Verantwortung für Lernen übernehmen.

- Wissen und Kompetenzen in Kommunikation mit anderen generieren.

- Lernverhalten vom Konsumieren zum Generieren verändern.

- Offenheit entwickeln.

\section{Digitale Strukturen}

Digitale Perspektive in Entwicklungs- und Strukturplänen präzisieren.

Profilbildung und Kooperationen entwickeln.
- Umsetzung einer digitalen Strategie mit konkreten Maßnahmen unterfüttert.

- Qualität der Digital-Konzepte durch die Vereinbarung konkreter Ziele stärken.

- Profilbildung und Kooperationen innerhalb der Hochschule oder mit anderen Hochschulen anvisieren bzw. vereinbaren.

- Anreize für profilbildende Kooperationen schaffen.

- Anreizsysteme für digital angereicherte Lehre entwickeln.

- Neue Lehrformate konzipieren und erproben. 


\begin{tabular}{|c|c|}
\hline $\begin{array}{l}\text { Veränderungsprozesse mit Blick auf Digitali- } \\
\text { sierung gestalten. }\end{array}$ & $\begin{array}{l}\text { - Ausgestaltung als Chance und Herausforde- } \\
\text { rung betrachten. } \\
\text { - Hochschulleitung adressieren. } \\
\text { - Veränderungsprozesse aktiv gestalten. } \\
\text { - Neues Rollenverständnis von Lehrenden und } \\
\text { Lernenden moderieren. } \\
\text { - Neue Professionsprofile in der Entwicklung } \\
\text { der Lehre gestalten. } \\
\text { - Moderne Infrastruktur bereitstellen. } \\
\text { - Neue Finanzierungsmodelle entwickeln. } \\
\text { - Anrechnung digitaler Lehre auf das Lehrde- } \\
\text { putat in den Fokus nehmen. } \\
\text { - Fokus auf Kompetenzorientierung mit Blick } \\
\text { auf innovative Lehr- und Lernszenarien rich- } \\
\text { ten. } \\
\text { - Raum und Anreize für Innovationen schaf- } \\
\text { fen. } \\
\text { - Bottom-up- und Top-down-Prozesse initiie- } \\
\text { ren und gemeinsam umsetzen. }\end{array}$ \\
\hline $\begin{array}{l}\text { Verbesserung der Schnittstellen gewähr- } \\
\text { leisten. }\end{array}$ & $\begin{array}{l}\text { - Gesamtübersicht von Learning-Management- } \\
\text { Systemen und Campus-Management-Syste- } \\
\text { men erstellen. } \\
\text { - Technische Schnittstellen identifizieren. } \\
\text { - Zusammenarbeit der beteiligten Akteurin- } \\
\text { nen und Akteure fördern. } \\
\text { - Durchdringung digitaler Strukturen auf allen } \\
\text { Ebenen steuern: Studium, Forschung, Lehre } \\
\text { und Verwaltung. } \\
\text { - Strukturelle Barrieren beim Übergang von } \\
\text { Lehre zur Forschung abbauen. } \\
\text { - Das Zusammenspiel zwischen Lehre, For- } \\
\text { schung und Administration effizient, aber } \\
\text { auch effektiv abbilden. } \\
\text { - Stärkere Integration digitaler Technologien } \\
\text { in der Lehre als zentrale Aufgabe betrach- } \\
\text { ten. }\end{array}$ \\
\hline
\end{tabular}


IT-Infrastruktur als zentrale Stellschraube in den Blick nehmen.

IT-Strukturen zwischen Zentralisierung und Dezentralisierung austarieren.

Campus-Management-Systeme als zentrale Instrumente integrieren.

E-Learning in den Fokus rücken.
- Informationstechnologie als einen unverzichtbaren Bestandteil nahezu aller Arbeitsabläufe an Hochschulen betrachten.

- IT-Management an Hochschulen stärken und zukunftsgerichtet aufstellen.

- Strategie, Governance, Portfolio und Budget für die IT-Infrastruktur zusammenhängend betrachten.

- Prioritäten und Qualitätskriterien festlegen; IT-Sicherheit und Datenschutz mitdenken.

- Verantwortlichkeiten zwischen dezentraler und zentraler IT-Versorgung benennen.

- Gemeinsame, regelmäßige Zusammenstellung und Entwicklung des IT-Portfolios als Bestandteil der IT-Strategie an Hochschulen betrachten.

- Chief Information Officer (CIO) etablieren.

- Effektive Weiterentwicklung unterstützen.

- Gegebenenfalls neues Campus-Management-System einführen.

- Anforderungen an das Projektmanagement, an die Kommunikation und an die effiziente Umsetzung der neuen (digitalen) Prozesse berücksichtigen.

- Projektmanagement und IT-Projektmanagement als langfristige Aufgabe erkennen.

- Entsprechende Ressourcen (Personal/Zeit/ Schulungen) einplanen.

- Enge Zusammenarbeit der Akteurinnen und Akteure (Verwaltung, IT, Fakultäten) fördern.

- Barrieren zwischen der Linienorganisation (Service) und der Projektorganisation reduzieren.

- Erprobung und Nutzung von modernen Lehrund Lernformaten möglichst hochschulweit denken.

- Finanzielle und personelle Anreize schaffen.

- Fehlende Kapazitäten ausgleichen.

- Diskurs über Didaktik fördern.

- Auseinandersetzung mit der Gestaltung innovativer Lehr- und Lernszenarien unter Einbezug digitaler Medien unterstützen.

- Good Practice-Beispiele durch Öffentlichkeitsmaßnahmen sichtbar machen. 
Aus der Perspektive der jeweiligen Akteurinnen und Akteure ergeben sich folgende Herausforderungen:

\section{Hochschulleitung}

- Veränderungsprozesse und Innovationsbereitschaft als Siegel der Hochschule deklarieren.

- Finanzierung ermöglichen.

- Lehrdeputat anpassen.

- Konzepte für eine gemeinsame digitale Infrastruktur vorantreiben.

- Digitalisierungsprozesse mit allen beteiligten Akteurinnen und Akteuren an Hochschulen abstimmen.

\section{Organisationseinheiten}

- Hardware- und Softwareinfrastruktur bereitstellen.

- Pflege, Wartung (inkl. Modernisierung) priorisieren.

- Support-Strukturen aufbauen und weiterentwickeln.

- Innovationsbereitschaft zeigen, neue Trends aufspüren.

- Anforderungen von Lehrenden und Studierenden an den Einsatz von Technik in den Blick nehmen.

\section{Hochschullehrende}

- Neuen Entwicklungen gegenüber aufgeschlossen sein, um kreative Lösungen umzusetzen.

- Veränderungsprozesse und Innovationsbereitschaft als Chance begreifen.

- Bei allen Veränderungen immer die Studierenden im Blick behalten.

\section{Studierende}

- Studienverlauf (Student-Life-Cycle) vereinfachen.

- Lehr- und Lernprozesse durch digital zur Verfügung stehende Inhalte mitgestalten.

- Kompetenzen im Umgang mit neuen Lehr- und Lernmethoden und den zugehörigen IT-Strukturen aufbauen. 


\section{Physische Lehr- und Lernräume}

Raumgestaltung als strategischen Fokus erkennen.
- Hochschulstrategie zur Lernraumentwicklung gestalten.

- Steigende Studierendenzahlen in den Blick nehmen.

- Neue didaktische Konzepte berücksichtigen.

- Digitale Herausforderungen bearbeiten.

Lehr- und Lernumgebungen an Lernbedürfnissen und Lernprozessen orientieren.

- Lernbedürfnissen und Lernprozessen der Studierenden bei der Entwicklung von digitalen und analogen Lehr-Lern-Settings in den Fokus rücken.

- Aufenthaltsqualitäten fokussieren.

- Vielfältige Lehr- und Lernraumangebote zur Verfügung stellen.

- Kooperation, Kreativität und Identifikation im Studium befördern.

- Hochschulspezifische Faktoren berücksichtigen.

- Zonierungen sowie flexible Raum- und Möblierungselemente nutzen.

- Unterschiedliche Nutzungsanforderungen berücksichtigen.

- Innovative und explorative Konzeptelemente wie Makerspaces oder Learning Labs erproben.

- Anpassung an sich verändernde Bedürfnisse vornehmen.

Zugang zu Lehr- und Lernräumen verbessern. - 24/7-Lernräume einrichten.

- Informationssysteme und Lernraumübersichten für die gesamte Hochschule erstellen.

- Raumbuchungssysteme anbieten.

Hybride Raumstrukturen entwickeln.
- Hybride Angebote ausbauen.

- Elektronische Dienstleistungen in den physischen Raum integrieren.

- Im virtuellen Raum auf physischen Raum verweisen.

- Schnittstellen von digitaler und physischer Lernwelt nutzen.

- Verschiedene Interaktionskonzepte unter Berücksichtigung des jeweiligen Raumkonzepts erstellen. 
Hochschulweite Entwicklung von Raumkonzepten implementieren.
- Lehr- und Lernraumkonzepte als strategisches Thema einstufen.

- Experimentierräume in Fakultäten und in zentralen Einrichtungen einrichten.

- Hochschulstrukturen zur sytematischen Entwicklung von Raumkonzepten schaffen bzw. vorhandene ausbauen.

Aus der Perspektive der jeweiligen Akteurinnen und Akteure ergeben sich folgende Herausforderungen:

\section{Hochschulleitung}

- Hochschulstrategisches Konzept in Zusammenarbeit mit allen an Lernraumplanung beteiligten Personen erarbeiten.

- Finanzressourcen für die Umsetzung von physischen Lernräumen bereitstellen.

- Nicht nur standardisierte Lernräume zur Verfügung stellen, sondern auch innovative Lehrund Lernraumlösungen anstreben.

- Strukturen und Personalressourcen für die Lernraumentwicklung einrichten.

\section{Organisationseinheiten}

- Neue Lernraumkonzepte gestalten und Mut beweisen, Lernraumkonzepte anders zu denken.

- Neue Lernraumkonzepte bewerben.

- Marketingkonzept entwickeln.

- Kooperationen über Organisationsgrenzen hinweg initiieren.

\section{Hochschullehrende}

- Bereitschaft zum experimentellen Arbeiten zeigen.

- Infrastrukturen in neu eingerichteten Lernräumen nutzen.

- Lehre in Verbindung mit dem selbstbestimmten Lernen gestalten.

- Mit verschiedenen Serviceeinrichtungen in der Hochschule kooperieren. 


\section{Studierende}

- Neu geschaffene Infrastrukturen für experimentelles Arbeiten und für Gruppenarbeit ausprobieren.

- In Gremien und Projekten zur Lernraumentwicklung engagieren.

- Verantwortung und Teilhabe für Lernraumentwicklungen übernehmen.

\section{Zukunft gestalten}

\section{Hochschulpolitik}

\section{Lernwelt Hochschule aufeinander abstimmen}

Deutschland profitiert von der föderalen Struktur, ermöglicht diese Struktur doch eine bessere Passung zwischen regionalen Bedarfen und der Steuerung der Regionen. Die daraus resultierende Uneinheitlichkeit der Gesetzgebungen und der Vereinbarungen der Länder mit den Hochschulen birgt eben dieses Potential, doch es erschwert gleichzeitig den Transfer von Good Practice. Hier gilt es seitens der Hochschulpolitik, das richtige Maß zwischen Vielfalt und Gleichartigkeit zu finden, um zum einen die Qualität des Studiums im ganzen Land verbindlich auf einem qualitativ hohen Niveau zu halten und die Vergleichbarkeit zu gewährleisten, zum anderen, um die Mobilität der Studierenden bei einem Wechsel des Bundeslandes im Rahmen ihres Studiums nicht zu erschweren. So empfehlen Babyesiza und Berthold die Zusammenführung von Vereinbarungen und Verträgen, um „ein Steuerungsmodell, das leicht vermittelt werden kann, und dessen Steuerungsimpulse sich nicht gegenseitig aufheben“ (Babyesiza/Berthold 2018, 34). Dies gilt auch für die Hochschulautonomie. Dabei sollte den Hochschulen genug Handlungsspielraum zur Entfaltung gegeben, aber gleichzeitig die Einhaltung der gesellschaftlichen Verpflichtungen der Hochschulen im Blick behalten werden. Vor diesem Hintergrund wird vor allem Ausweitungsbedarf bei den Themen Kapazitätsverordnung, Betreuungsverhältnis, tarifliche Flexibilisierung, Infrastrukturentwicklung und Public-Private-Partnership gesehen (Weichert/Stang 2020, 169). 


\section{Einheitliche Struktur von Entwicklungs- und Strukturplänen (ESPs) sowie Zielvereinbarungen schaffen}

Da es keine einheitliche Struktur von Entwicklungs- und Strukturplänen (ESPs) sowie Zielvereinbarungen gibt, erschwert dies die Vergleichbarkeit der Rahmenbedingungen der Gestaltung der Lernwelt Hochschule und die Aufstellung von Benchmarks. Um bundesweit vergleichbare Strukturen zu schaffen, bedarf es einer Angleichung des Aufbaus von Entwicklungs- und Strukturplänen. Auch wäre es hilfreich, wenn Zielvereinbarungen als individuelle Aushandlung zwischen dem jeweiligen Landesministerium und den Hochschulen (Weichert 2020) in ihrer Struktur vergleichbar und öffentlich zugänglich wären. Ein bundesweiter Orientierungsrahmen für die Gestaltung der Lernwelt Hochschule wäre hilfreich, um die Zielrichtungen besser vergleichen zu können. Es geht dabei weniger um die Vereinheitlichung, sondern um eine verstärkte Transparenz. Die Hochschulautonomie wäre dadurch nicht beeinträchtigt.

\section{Zielvereinbarungen als Stellschrauben nutzen}

Um die Lernwelt Hochschule ganzheitlich $\mathrm{zu}$ betrachten, bedarf es einer verstärkten Vernetzung der einzelnen Akteurinnen und Akteure, die an Hochschulen von Relevanz sind, wie unter anderen Hochschulleitung, Hochschulverwaltung, IT- und Infrastrukturabteilung, Bibliothek, Didaktikzentrum und besonders die Lehrenden und Studierenden. Im Rahmen von Zielvereinbarungen könnte ein Fokus auf dieser Vernetzung liegen. Dabei könnte eine Prioritätensetzung seitens der Hochschule ermöglicht werden. Es müssten allerdings Kriterien zur Bewertung der Vernetzungsaktivitäten festgelegt werden.

\section{Unterschiedliche Steuerungsmodelle berücksichtigen}

Die Hochschulpolitik sollte den Rahmen schaffen, der verschiedene Steuerungsmodelle darstellbar macht. Dabei sollte die Studierendenorientierung im Fokus der Steuerungsmodelle stehen. So ist die derzeitige Anreizstruktur, die vor allem die eingeworbenen Drittmittel im Blick hat, nicht dazu geeignet, die Entwicklung der Lehre zu befördern. Es gilt den Wandel von der organisationsfokussierten Perspektive zur studierendenorientierten Perspektive zu lancieren. Hierfür müssen Mittel und Methoden verfügbar gemacht werden, die dies ermöglichen. 


\section{Selbstorganisation optimieren}

Die Hochschulautonomie ist ein wichtiges Gut und für die Gestaltung einer vielfältigen Hochschullandschaft unerlässlich. Will man allerdings die Studierendenorientierung stärker als Leitmotiv von Handlungskoordination von Hochschulen etablieren, bedarf es zunächst auch Studien zur Studierendenorientierung, um Bedarfe und Möglichkeiten, aber auch schon bestehende Good Practice systematisch zu erfassen und in die Lernwelt Hochschule zu transferieren. Es sollte auch Orientierung gegeben werden, wie in Hochschulen - jenseits teilweise formalstatischer Gremien - Austauschmöglichkeiten für alle Akteurinnen und Akteure geschaffen werden können. Dabei geht es vor allem darum, dass sich die Akteurinnen und Akteure über Fach- und Sachbezüge hinweg besonders über die Schnittstellen und Verbindungen ihrer Kernexpertise zu anderen Themen - austauschen können. Hochschulpolitik sollte hier Orientierungen liefern.

\section{Finanzierungssituation verbessern}

Um die notwendigen Entwicklungen vorantreiben zu können, bedarf es einer langfristig ausgelegten Förderung, da dieser grundlegende Wahrnehmungswandel der Lernwelt Hochschule historisch gewachsene Vorstellung bricht und sowohl die Strukturen wie auch die Prozesse der Organisation Hochschule in allen Bereichen verändern wird. Der Shift from Teaching to Learning (Barr/Tagg, 1995) spielt in die Hochschulpolitik hinein und erfordert zusätzliche - auch finanzielle Anstrengungen - der Bundesländer. Dabei geht es um die langfristige Sicherung von Innovationen in der Lernwelt Hochschule. Dazu bedarf es neuer Finanzierungskonzepte.

\section{Bologna-Prozess weiterentwickeln}

Der Perspektivwechsel zur Studierendenorientierung tangiert auch die BolognaReform, die auf Ergebnisse (Outcomes) und Kompetenzen abzielt, indem sich die Prozesse ändern müssen, wie diese Outcomes zustande kommen, Prüfungsformen überdacht werden müssen, da sich „trotz umfassender Bemühungen, [...] die erwünschten Effekte [...] nicht eingestellt haben“ (Brahm et al. 2016, 25). Die Perspektive der Kompetenzorientierung sollte konsequent weiterverfolgt und traditionelle Leistungsmessungsstrukturen, die sich durch eine starke Klausurenorientierung auszeichnen, kontinuierlich reduziert werden. Hier be- 
darf es auch von Seiten der Hochschulpolitik klarer Hinweise, ohne die Freiheit von Forschung und Lehre einzuschränken.

\section{Reformprozesse fördern}

Betrachtet man die Entwicklung der Hochschullandschaft in Deutschland, wird deutlich, dass zwar in vielen Teilbereichen an Reformen gearbeitet wird, es aber keine grundlegenden Reformprozesse in Bezug auf die Lernwelt Hochschule gibt. Auch wenn hier einiges in den letzten Jahren angestoßen wurde, gibt es einen erhöhten Bedarf, die Lernwelt Hochschule in der Gesamtheit zu reformieren. Für Hochschulpolitik - auf Länder- und Bundesebene - gilt es, ganzheitliche Innovationskorridore zu entwickeln, in deren Rahmen Hochschulen ihren Weg finden können. Allerdings wird es ohne intensives Engagement der Hochschulen schwierig, die deutschen Hochschulen international konkurrenzfähig zu machen. Hier bedarf es konzertierter Aktivitäten.

\section{Transparente Datenstruktur erzeugen}

Die Perspektive der Studierendenorientierung ist bislang nicht im Fokus der Hochschulforschung. Vor diesem Hintergrund ist es erstrebenswert, eine Datenbasis zu schaffen, in der diese Perspektive transparent dokumentiert wird und die Daten zugänglich macht. Hier würde schon helfen, wenn Entwicklungsund Strukturpläne sowie Zielvereinbarungen gebündelt vorliegen würden. Auch eine Kartierung bezogen auf Lern-Management-Systeme oder physische Lehr- und Lernräume würde dabei unterstützen, Problembereiche zu erkennen und bearbeiten zu können. So könnten effektivere und effizientere Lösungen gefunden werden sowie doppelte Aufwände und Fehlversuche reduziert oder ganz vermieden werden. Dazu bedürfte es allerdings einer bundesweit agierenden Institution.

\section{Entwicklung dynamisieren}

Da Deutschland in Europa im Kontext der innovativen Gestaltung von Hochschulentwicklung eher zu den „latecomern“ gehört (Becker/Stang 2020a), bedarf es verstärkter Anstrengungen, hier aktiv zu werden. Hochschulpolitische Entscheidungsprozesse müssten effektiviert und beschleunigt, bürokratische Hindernisse aus dem Weg geräumt und die traditionelle Hochschulkultur grund- 
legend verändert werden. Vor allem staatliche Hochschulen benötigen ein verändertes Innovationsklima. Dieses Klima muss durch die Hochschulpolitik geschaffen werden. An einzelnen Hochschulen werden äußerst innovative Wege begangen, oft unter Nichtberücksichtigung hemmender bürokratischer Strukturen. Diese bürokratischen Strukturen müssen auf ein Minimum reduziert werden.

\section{Good Practice ${ }^{2}$}

- Bundesländer: Die Wissenschaftsministerien der Bundesländer setzen die Rahmenbedingungen und fördern Projekte ${ }^{3}$.

- Bundesministerium für Bildung und Forschung (BMBF): Diverse Projekte zur Entwicklung der Hochschullandschaft ${ }^{4}$.

- Bund und Bundesländer: Vereinbarungen wie zum Beispiel beim Qualitätspakt Lehre ${ }^{5}$ oder dem Hochschulpakt ${ }^{6}$.

- Centrum für Hochschulentwicklung (CHE): Wirft den Blick auf ein leistungsstarkes und faires Hochschulsystem und liefert Studien und Rankings ${ }^{7}$.

- Deutsche Initiative für Netzwerkinformation e. V. (DINI): Möchte die Informations- und Kommunikationsdienstleistungen und die dafür notwendige Entwicklung der Informationsinfrastrukturen an den deutschen Hochschulen fördern, u. a. auch bezogen auf die Gestaltung von Lernräumen ${ }^{8}$.

- European University Association (EUA): Vertritt über 800 Hochschulen in Europa und liefert Studien und Übersichten zu Good Practice?.

- Hochschulforum Digitalisierung (HFD): Versteht sich als Think Tank, der den Diskurs zur Hochschulbildung im digitalen Zeitalter fördert und Studien und Arbeitspapiere für die Hochschulpraxis zur Verfügung stellt ${ }^{10}$.

2 Die Hinweise im Bereich Good Practice sind Ergebnisse der Recherchen im Rahmen des Projektes Lernwelt Hochschule. Dabei konnten sicher nicht alle Good Practice-Beispiele berücksichtigt werden. Es geht hier um Hinweise, die sicher noch ergänzt werden können.

3 https://www.kmk.org/kmk/mitglieder.html.

4 https://www.bmbf.de/de/studium-70.html.

5 https://www.bmbf.de/de/qualitaetspakt-lehre-524.html.

$6 \mathrm{https} / / /$ www.bmbf.de/de/hochschulpakt-2020-506.html.

7 https://www.che.de/.

8 https://dini.de/.

9 https://eua.eu/.

$10 \mathrm{https}$ //hochschulforumdigitalisierung.de/de. 
- Hochschulinformationssystem e. G. (HIS): Liefert Daten zur Hochschulplanung und -entwicklung ${ }^{11}$.

- Hochschulrektorenkonferenz (HRK): Versteht sich als Repräsentanz der Hochschulen und als institutionelles Zentrum des deutschen Wissenschaftssystems ${ }^{12}$.

- Kultusministerkonferenz (KMK): Sieht ihre Aufgabe darin, durch Konsens und Kooperation für die Lernenden, Studierenden, Lehrenden und wissenschaftlich Tätigen das erreichbare Höchstmaß an Mobilität zu sichern ${ }^{13}$.

- Stifterverband für die Deutsche Wissenschaft e.V.: Bringt Unternehmen und Hochschulen zusammen, vergibt Preise und veröffentlicht Studien und liefert einen Überblick über Good Practice ${ }^{14}$.

\section{Hochschulorganisation}

\section{Gesamtstrategie in den Blick nehmen}

Hochschulintern ist es von großer Bedeutung, alle Akteurinnen und Akteure zusammenzubringen, die Hochschule ganzheitlich zu denken, einzelne Lösungen miteinander zu verweben, Querschnittsstellen einzurichten und die Information und Kommunikation in der gesamten Hochschule zu verstetigen. Dazu gehört auch, die Beteiligung aller Hochschulakteurinnen und -akteure sicherzustellen, indem feste Strukturen und Prozesse geschaffen werden, die über „Arbeitsgruppen“ und „projektinterne Kommunikation“ (Becker/Stang 2020b) hinausgehen. Dabei sind entscheidungsfähige Gremien nur ein Weg, die Zusammenarbeit der Beteiligten $\mathrm{zu}$ intensivieren. Hier gilt es Kooperationsmodelle $\mathrm{zu}$ entwickeln, die alle Interessensgruppen berücksichtigen. Wichtig ist es dabei, die Gesamtstrategie nicht aus den Augen zu verlieren.

\section{Ungleichgewicht von Forschung und Lehre beseitigen}

Die derzeitige Schieflage in der Bewertung von Forschung und Lehre im deutschen Hochschulsystem liegt unter anderem darin begründet, dass es für For-

$\overline{11}$ https://www.his.de/willkommen.html.

12 https://www.hrk.de/.

13 https://www.kmk.org/themen/hochschulen.html.

$14 \mathrm{https} / / /$ www.stifterverband.org/. 
schung und den daraus resultierenden Publikationen und Drittmitteln Gratifikationen für die Hochschulen und für die Forschenden gibt. Es gilt für die Zukunft den Stellenwert von Lehre nicht nur über Preise für gute Lehre deutlich zu machen, sondern auch hier ein Gratifikationssystem zu entwickeln, das Lehrexzellenz auch monetär für die Hochschulen und die Lehrenden auszeichnet. Hierzu gilt es auch Kriterienraster zu entwickeln, die als Orientierung zur Bewertung exzellenter Lehre dienen können. Lehre soll nicht nur zur Qualitätssicherung, sondern auch zu einer besseren Reputation der Hochschule beitragen.

\section{Strategische Hochschulentwicklung gestalten}

Hochschulen sind in vielen Bereichen gekennzeichnet von einem traditionellen Verständnis, wie Hochschulen gestaltet sein sollen, wie zum Beispiel Fakultätsstrukturen, Orientierung der Lehre etc. Um den Anforderungen bezüglich der Bewältigung zukünftiger Herausforderungen der Gesellschaft gerecht zu werden, bedarf es allerdings einer veränderten Strategie bei der Hochschulentwicklung. Die Öffnung der Hochschule auch im Kontext Public Private Partnership (PPP) hat in den letzten Jahren zugenommen und wird auch im Ausland intensiviert (Weichert/Stang 2020). Eine solche Öffnung ermöglicht es, die Lernwelt Hochschule so zu gestalten, dass Projekte mit realer Aufgabenstellung in Partnerschaft mit Wirtschaftsunternehmen oder Institutionen studiengangübergreifend und interdisziplinär bearbeitet werden können. Dabei muss allerdings gewährleistet sein, dass keine Abhängigkeit in der Lehre von den Unternehmen oder Institutionen entsteht. Die Strukturen und Prozesse bei der Zusammenarbeit müssen deshalb so geregelt werden, dass die Freiheit von Lehre nicht eingeschränkt wird. Der Gestaltung der Hochschulstrategie im Netzwerk aktueller und potentieller Stakeholder kommt dabei eine besondere Bedeutung zu.

\section{Leitbilder sowie Entwicklungs- und Strukturpläne präzisieren}

Leitbilder sowie Entwicklungs- und Strukturpläne sind die Basis für die strategische Ausrichtung in Bezug auf die Lernwelt Hochschule (Becker/Stang 2020b; Weichert 2020). Neben quantitativen Betrachtungen, wie anvisierte Studierendenzahlen, bedarf es einer verstärkten Berücksichtigung qualitativer Kriterien. Die Frage, wie Lehre an der Hochschule gestaltet sein soll und wie - unter der Perspektive der Studierendenorientierung - Lernen unterstützt werden soll, ist dabei zentral. Dies bedeutet, dass neben einem Leitbild Lehre auch ein Leitbild Lernen entwickelt wird, an dessen Formulierung alle Hochschulakteurinnen 
und -akteure beteiligt werden. Diese Leitbilder könnten dann die Basis für veränderte Entwicklungs- und Strukturpläne sein. Diese Dokumente könnten dann auch die Grundlage für die Zielvereinbarungen mit den jeweiligen Trägerinnen und Trägern darstellen.

\section{Beteiligung der Hochschulakteurinnen und -akteure sicherstellen}

Durch die Hochschulgesetzgebung vorgegebene Gremien bilden die formale Beteiligungsstruktur von Hochschulen. Dabei werden nur ausgewählte Hochschulakteurinnen und -akteure berücksichtigt. Um die Entwicklung der Hochschule auf eine breite Basis zu stellen, bedarf es einer stärkeren Partizipation der Organisationsmitglieder der Hochschule. Vor allem die Einbindung von Studierenden in strategische Entscheidungsbildungsprozesse der Hochschule stellt dabei eine große Herausforderung dar. Die Entwicklung eines Partizipationsmodells gehört zu den zentralen Elementen einer veränderten Hochschulorganisation. Hier werden in Zukunft neue Wege gestaltet werden müssen, wie zum Beispiel die Einbindung von Studierenden auf Hochschulleitungsebene.

\section{Zusammenarbeit der Hochschulakteurinnen und -akteure intensivieren}

Die Organisationseinheiten von Hochschulen agieren oft noch getrennt voneinander (Becker/Stang 2020b). Um die Hochschule als ganzheitliche Lernwelt Hochschule zu entwickeln, bedarf es einer stärkeren Vernetzung der verschiedenen Verwaltungsabteilungen, Fakultäten, Institute sowie nicht zuletzt auch der Lehrenden und der Studierenden. Dabei geht es in erster Linie darum, die sehr unterschiedlichen Vorstellungen und Kulturen bezogen auf die Lernwelt Hochschule anzunähern und den Austausch zu fördern. Die Entwicklung eines Kooperationsmodells bezogen auf die verschiedenen Hochschulakteurinnen und -akteure und auch auf externe Stakeholder könnte hier ein Ansatz sein. Alleine bei der Entwicklung eines solchen Kooperationsmodells könnten Problembereiche, die bearbeitet werden müssten, sichtbar werden.

\section{Zugänglichkeit der Hochschule ermöglichen}

Die Zugangskonzepte bezogen auf Studierende sind in Hochschulen sehr unterschiedlich gestaltet. Dabei wird zum Beispiel die Zugänglichkeit der Lehr- und Lernräume und des Hochschulgebäudes teilweise sehr restriktiv - Lehrräume 
können nur im Kontext von Lehrveranstaltungen genutzt werden und sind sonst verschlossen - oder eben sehr liberal - der Zugang ist jederzeit möglich geregelt. Auch die Zugänglichkeit zu Services wie Bibliothek oder Technik- beziehungsweise Materialausleihe sind teilweise auch an einzelnen Hochschulen nicht einheitlich geregelt. Die Zugänge zu digitalen Services erfordern nicht selten unterschiedliche Anmeldeprozeduren (Becker/Stang 2020b). Die Entwicklung eines konsistenten Zugangskonzepts, das für alle Hochschulakteurinnen und -akteure transparent ist, kann hier eine Option sein. Dabei ist es hilfreich, wenn auch im Kontext digitaler Strukturen, ein Zugang zu allen Services über eine Kennung erfolgt und nicht unterschiedliche Anmeldeprozeduren erfordert.

\section{Hochschule als multidimensionale Organisation koordinieren}

Hochschule ist eine multidimensionale Organisation, die im Hinblick auf die Stellenstrukturen und Kompetenzen eine extrem hohe Komplexität aufweist. Diese Komplexität, die sich in den Logiken und Kulturen von Verwaltung, Forschung, Lehre, IT-Abteilungen, Infrastrukturabteilungen usw. findet, gilt es zu moderieren. Die Lernwelt Hochschule zeichnet sich zum Beispiel im Bereich der Lehre auch durch unterschiedliche Beschäftigungsmodi aus. So wird sie von verbeamteten oder nicht verbeamteten Professorinnen und Professoren, fest oder befristet angestellten Mitarbeitenden und externen Lehrbeauftragten realisiert. Um Hochschulorganisation in Bezug auf die Lernwelt Hochschule ganzheitlich zu gestalten, bedarf es hier einer verbesserten Koordination auch bezogen auf ein Leitbild Lehre, dem eine Studierendenorientierung zugrunde liegt. Die Lehrenden sollten hier eine grundlegende Orientierung erhalten, welche Überlegungen handlungsleitend sein könnten. Ähnliche Orientierungsstrukturen könnten für andere Hochschulbereiche geschaffen werden.

\section{Ganzheitliche und integrierte Lernwelt entwickeln}

Studierende nehmen die Hochschule oft als segmentiert und additiv wahr (Gläser/Kobsch 2020). Dies liegt unter anderem darin begründet, dass die Hochschule nicht ganzheitlich gesteuert und nicht studierendenorientiert gedacht wird. Die Beteiligung von Studierenden ist nur sehr gering ausgeprägt - hier werden zurzeit vereinzelt neue Wege erprobt (Becker/Stang 2020b, 89-90). Den Studierenden fehlt das Wissen über die Organisation Hochschule. Letztlich trägt die Studienstruktur mit der Ausrichtung auf Workload und ECTS dazu bei, dass den Studierenden der Anreiz fehlt, sich aktiv in die Entwicklung der Lehre oder 
grundsätzlich in die Organisation einzubringen. Hier könnten Maßnahmen wie die Verrechnung des Engagements mit einem Äquivalent zu den „social points“ Ansätze sein, die Studierendenperspektive mit in die Entscheidungsbildungsprozesse einzubringen. Entwicklungen im Ausland zeigen, dass ein studierendenorientierter Zugang dazu führt, dass alle Beteiligten von Innovationen profitieren (Weichert/Stang 2020).

\section{„Atmende“ Hochschule denken}

Insgesamt zeichnet sich ein Wandel der Strategien, Strukturen, Prozesse und Organisationskulturen mit dem Perspektivwechsel zur studierendenorientierten Hochschule ab. Die in dieser Hinwendung inkludierte Flexibilität der Organisation erfordert eine atmende Hochschule, die sich agil an die schnell wechselnden Herausforderungen aus ihren Umwelten anpassen kann. Diese Flexibilität kann durch flachere Hierarchien, die Strategieentwicklung mit allen Hochschulakteurinnen und -akteuren und eine breit angelegte Kommunikations- und Beteiligungskultur etabliert werden. Dabei sollte der Student-Life-Cycle in der Lernwelt Hochschule im Fokus der Überlegungen stehen.

\section{Good Practice}

- Code University Berlin: „Lernen auf Augenhöhe“, projektorientiertes Lernen usw. werden im Rahmen einer veränderten Organisationskultur umge$\operatorname{setzt}^{16}$.

- Fachhochschule Potsdam: Studierende werden mit dem Amt einer/s Studentische/n Vizepräsidenten/in in die Hochschulleitung integriert ${ }^{17}$.

- Hochschule der Medien Stuttgart: In der Fakultät „Information und Kommunikation“ wird eine Transdisziplinarität auch organisatorisch umgesetzt (Mildenberger/Vonhof 2020); mit LAPS (Learning Analytics für Prüfungsleistungen und Studienerfolge) sollen Studierende, insbesondere in der Studieneingangsphase, individuell unterstützt werden ${ }^{18}$.

15 Die Hinweise im Bereich Good Practice sind Ergebnisse der Recherchen im Rahmen des Projektes Lernwelt Hochschule. Dabei konnten sicher nicht alle Good Practice-Beispiele berücksichtigt werden. Es geht hier um Hinweise, die sicher noch ergänzt werden können.

16 https://code.berlin/de/.

17 https://www.fh-potsdam.de/informieren/organisation/hochschulleitung/.

$18 \mathrm{https} / / /$ www.hdm-stuttgart.de/laps. 
- Hochschule Osnabrück: Im LearningCenter werden Lehr-Lern-relevante Aspekte gebündelt ${ }^{19}$ und es besteht grundsätzlich die Möglichkeit, Abschlüsse in Teilzeit oder berufsbegleitend zu erlangen ${ }^{20}$.

- SRH Hochschule Heidelberg: Mit dem Studienmodell CORE (Competence Oriented Research and Education) wurden alle Studiengänge kompetenzorientiert ausgerichtet und lehrstrategische Gesamtausrichtung für die Hochschule zugrunde gelegt (Ninnemann et al. 2020) ${ }^{21}$.

- Universität Hamburg: Der Projektbereich Hochschullehre und studentische Partizipation versteht sich als Unterstützungsangebot zur Verbesserung von Studium und Lehre unter der Perspektive von Studierendenpartizipation ${ }^{22}$.

- Universität Hildesheim: Die Vizepräsidentin für Lehre und Studium pflegt das Prinzip der offenen Bürotür. Damit werden hierarchische und formelle Hürden abgebaut ${ }^{23}$.

- Universität Konstanz: Bietet ein Unterstützungsangebot für Lehrende und unterstützt alle digitalen Prozesse rund um die Lehre ${ }^{24}$.

- Universität Lüneburg: Studierende werden in Qualitätszirkeln einbezogen ${ }^{25}$ und das Studium Individuale schafft einen flexiblen Zugang für die Studierenden ${ }^{26}$.

- Universität Trier: Digitalisierung wird aus Ausgangspunkt für eine weitergehende Hochschulentwicklung genommen (Höfler-Hoang et al. 2020).

- Universität Twente: Das TOM-Modell basiert nicht nur auf didaktischen sondern auch organisatorischen Faktoren (University of Twente 2017) und stärkt damit die Lehre ${ }^{27}$.

- Technische Universität Hamburg: Im Projekt mytrack - individuelles Lernen in der Studieneingangsphase besteht für Studierende die Möglichkeit, sich besser im Studium zu orientieren, Ziele zu verfolgen, Zeit zum Lernen zu finden und einen eigenen Weg durch das Studium zu gehen (in Kooperation mit der Technischen Hochschule Mittelhessen und der Hochschule Fulda) ${ }^{28}$.

19 https://www.hs-osnabrueck.de/de/learningcenter/.

20 https://www.hs-osnabrueck.de/de/studium/studienangebot/berufsbegleitend-oder-berufsintegrierend-studieren/.

21 https://www.hochschule-heidelberg.de/de/core-prinzip/.

22 https://studpart.check.uni-hamburg.de/.

23 https://www.uni-hildesheim.de/organe-und-gremien/praesidium/.

24 https://www.uni-konstanz.de/lehren/beratung-hilfsmittel-service/digital-unterstuetzte-lehre/.

25 https://www.leuphana.de/lehre/feedback-zu-lehre-und-studium/qualitaetszirkel.html.

$26 \mathrm{https}$ //www.leuphana.de/college/bachelor/studium-individuale.html.

$27 \mathrm{https}: / /$ www.utwente.nl/en/tom/.

28 https://mytrack-tuhh.de. 


\section{Hochschuldidaktik}

\section{Shift from Teaching to Learning in den Fokus rücken}

Didaktische Ansätze, die problembasiertes, projektorientiertes und forschungsorientiertes Lernen in den Fokus rücken, spielen an Hochschulen eine zunehmend wichtigere Rolle. Dabei wird kollaboratives Arbeiten immer wichtiger. Die Rolle der Lehrenden verändert sich in Richtung Lernbegleitung. Ziel dabei ist es, Problemlösekompetenz zu vermitteln und die Aneignung von Wissen durch selbstgesteuertes Lernen zu etablieren. Um einen solchen Zugang zu realisieren, bedarf es veränderter Lehr-Lernszenarien, in denen auch die Lehrenden-Lernenden-Hierarchie aufgehoben wird. Dabei müssen sich auch Studierende von der Konsumhaltung verabschieden. Um die Rahmenbedingungen für solche didaktischen Konzeptionen zu schaffen, sind auch veränderte digitale Strukturen und Lehr-Lernraumarrangements notwendig. Auch die Struktur von Stundenplänen muss überdacht und Wissenserwerb stärker in kontextualisierten Blockangeboten verankert werden.

\section{Verändertes Rollenverständnis der Lehrenden etablieren}

Lehrende haben die Aufgabe, den Prozess des Lernens zu organisieren, zu initiieren und zu begleiten. Dabei geht es um die Gestaltung des didaktischen Rahmens, der Lernenden die Möglichkeit bietet, ihr Lernen gestalten zu können. Dies erfordert allerdings auch eine große Verantwortung der Lernenden für ihr Lernen. Lehre wird so zur Gestaltung eines Ermöglichungsraums. Dies erfordert von den Lehrenden eine hohe Flexibilität und pädagogische Expertise, die an Hochschulen bislang nur rudimentär vorhanden ist. Lehr-Lernprozesse werden zu Kommunikationsprozessen. Dabei wird der Vielfalt der Zugänge, Lernwege und Ergebniskonstruktionen Rechnung getragen. Dies erfordert einen grundlegenden Perspektivwechsel auf Seiten der Lehrenden. Dazu müssen Fortbildungsaktivitäten verstärkt und Experimentierräume für innovative Lehre etabliert werden.

\section{Wissensproduktion und Kompetenzentwicklung neu gestalten}

Lernen findet durch gemeinsame Generierung von Wissen statt. Wissensgenerierung durch den Konsum von Information ist nur begrenzt möglich. Wissen 
und Kompetenzen werden in Kommunikation mit anderen generiert beziehungsweise entwickelt. Die Entwicklung geht vom Konsumieren zum Generieren. Durch die digitalen, mobilen Medien wird diese Entwicklung beschleunigt. Um die Lernoptionen an Hochschulen weiterzuentwickeln, bedarf es einer strategischen Gestaltung von Methodenvielfalt. Die Entwicklung eines Methodenportfolios, aus dem sich die Lehrenden bedienen können, könnte hier eine Grundlage bilden. Bislang entstehen methodische Innovationen eher punktuell und es gibt selten einen Überblick, wer mit welchen Methoden arbeitet. Eine solche hochschulspezifische Methodenübersicht könnte dazu dienen, Innovationen in der Lehrenden-Community zu verbreiten. Die Offenheit der Lehrenden, aber auch der Studierenden, ist allerdings erforderlich, um solche Methodeninnovationen erfolgreich umzusetzen.

\section{Didaktikzentren als Schlüsseleinrichtungen anerkennen}

Die Didaktikzentren haben die Aufgabe, Lernende und Lehrende unter anderem methodisch aus- und weiterzubilden. Weitere Aufgaben können die Gestaltung von Lehr- und Lernmedien, Supervision der Einzelstudierenden und Kleingruppen, Lern- und Lehrcoaching, Beratungskompetenzen der Lehrenden entwickeln und die Vermittlung didaktischer Kompetenzen sein. Unter der Perspektive veränderter Lehr-Lernszenarien wird ihre Bedeutung noch wachsen. Es wird nicht ausreichen, die Didaktikzentren nur als Projekte in den Hochschulen zu verorten. Die Hochschulen werden nicht umhinkommen, diese als zentrale Schlüsseleinrichtung zu etablieren. Sie können auf konzeptioneller Ebene als fachübergreifende Verteilerinnen für Innovation in der Lehre, als Unterstützerinnen der Curriculum-Entwicklung und des Qualitätsmanagements Lehre fungieren. Deshalb sollten sie verstetigt und in der Hochschulorganisation verankert werden, um ihre Schnittstellenfunktion zu den relevanten Abteilungen (ITAbteilung, Infrastruktur-Abteilung etc.) wahrnehmen zu können.

\section{Leitbild Lehre als Qualitäts- und Kulturmerkmal positionieren}

Auch wenn der Blick auf die Lehre vor dem Hintergrund von Aktivitäten wie der Exzellenzinitiative des Bundes teilweise in den Hintergrund geraten ist, wird bei der Betrachtung der Bedeutung der Lernwelt Hochschule für die zukünftige Entwicklung der Gesellschaft deutlich, dass der Bereich der Lehre wieder stärker in den Fokus gerückt werden muss. Auch wenn vielfältige Projektinitiativen unter anderem durch das Bundesministerium für Bildung und Forschung 
(BMBF) hier in den letzten Jahren wichtige Impulse gesetzt haben, bleibt in den Hochschulen noch viel zu tun. Ein explizites Leitbild für die Lehre kann hier für den hochschulinternen Diskurs von besonderer Bedeutung sein. Dabei müssen allerdings alle Hochschulakteurinnen und -akteure einbezogen werden. Mit einem solchen Leitbild kann ein Bewusstsein für gute Lehrqualität entwickelt werden, nicht nur bei den Lehrenden, sondern auch bei den Studierenden, die oft nicht wissen, was sie von der Lehre erwarten dürfen. Ein solches Leitbild kann auch für die Außendarstellung verwendet werden. Studieninteressierte können sich ein Bild davon machen, welches Lehr-Lernkonzept an der jeweiligen Hochschule zugrunde liegt. Idealerweise wird dieses Lehr-Lernkonzept von allen Lehrenden mitgetragen.

\section{Good Practice $^{29}$}

- Brandenburgische Technische Universität Cottbus-Senftenberg (BTU): Genius Loci-Preis für Lehrexzellenz $2019^{30}$.

- Bundesministerium für Bildung und Forschung (BMBF): Projektdatenbank zum Qualitätspakt Lehre ${ }^{31}$.

- Code-University Berlin: Bietet „Lernen auf Augenhöhe“ und erfahrungsbasiertes Studium ${ }^{32}$.

- Deutsche Gesellschaft für Hochschuldidaktik: Wissenschaftliche Fachgesellschaft aller an den Themen „Hochschuldidaktik“ und „Studienreform“ interessierten Personen im deutschsprachigen Raum ${ }^{33}$.

- Duale Hochschule Baden-Württemberg: Hat lehrintegrierte Forschung als strategisches didaktisches Handlungsfeld verankert (Kuhn et al. 2020).

- Fachhochschule Potsdam: Mit \#DiLehre soll eine bedarfsorientierte Entwicklung einer nachhaltigen organisatorischen Struktur für die Digitalisierung in der Lehre geschaffen werden ${ }^{34}$.

29 Die Hinweise im Bereich Good Practice sind Ergebnisse der Recherchen im Rahmen des Projektes Lernwelt Hochschule. Dabei konnten sicher nicht alle Good Practice-Beispiele berücksichtigt werden. Es geht hier um Hinweise, die sicher noch ergänzt werden können.

30 https://www.b-tu.de/.

31 https://www.qualitaetspakt-lehre.de/de/massnahmen-und-themenfelder-der-gefordertenprojekte-suchen-und-finden-1745.php.

32 https://code.berlin/de/concept/.

33 https://www.dghd.de/.

34 https://www.fh-potsdam.de/informieren/service/e-learning/projekte/projekt-detailansicht/ project-action/digitalisierung-der-lehre/. 
- Hochschule der Medien Stuttgart: Schlüsselkompetenzen erhalten einen zentralen Status in der Lehre (Burmester/Seidl 2020).

- Hochschule für angewandte Wissenschaften Coburg: Genius Loci-Preis für Lehrexzellenz $2019^{35}$.

- Leibniz-Institut für Wissensmedien: Das Informationsportal e-teaching.org bietet wissenschaftlich fundierte Informationen $\mathrm{zu}$ didaktischen, technischen und organisatorischen Aspekten von E-Learning ${ }^{36}$.

- $\quad$ RWTH Aachen: Genius Loci-Preis für Lehrexzellenz $2017^{37}$.

- SRH Hochschule Heidelberg: Genius Loci-Preis für Lehrexzellenz 2018 ${ }^{38}$.

- Technische Hochschule Köln: Mit dem Zentrum für Lehrentwicklung werden vielfältige Angebote zur Verbesserung von Lehren und Lernen zur Verfügung gestellt ${ }^{39}$ und Genius Loci-Preis für Lehrexzellenz $2017^{40}$.

- Technische Hochschule Nürnberg Georg Simon Ohm: Mit dem Learning Lab wird für Lehrende und Studierende ein Experimentierraum bereitgestellt ${ }^{41}$.

- Technische Universität München: ProLehre / Medien und Didaktik bündelt vielfältige hochschul- und mediendidaktische Kompetenzen und verknüpft dabei wissenschaftliche Erkenntnisse der Lehr- und Lernforschung mit dem Erfahrungswissen der Hochschullehrenden ${ }^{42}$.

- Universität Düsseldorf: Bietet studentische Online-Partizipation zur Mitgestaltung von Seminarinhalten ${ }^{43}$.

- Universität Erlangen-Nürnberg: Das Institut für Lern-Innovation bietet als E-Learning-Zentrum und Forschungsinstitut Dienstleistungen, Beratung und Know-How-Transfer an ${ }^{44}$.

- Universität Halle-Wittenberg: Das Zentrum für multimediales Lehren und Lernen (@LLZ) soll digitale Hochschulbildung durch Beratung und Unterstützung, Evaluation und Forschung gestalten ${ }^{45}$.

35 https://www.hs-coburg.de/.

36 https://www.e-teaching.org/.

37 https://www.rwth-aachen.de/.

38 https://www.hochschule-heidelberg.de/.

39 https://www.th-koeln.de/hochschule/zentrum-fuer-lehrentwicklung_47876.php.

40 https://www.th-koeln.de/.

$41 \mathrm{https}$ //www.th-nuernberg.de/einrichtungen-gesamt/abteilungen/service-lehren-und-lernen/learning-lab/.

42 https://www.prolehre.tum.de/home/.

43 https://diid.hhu.de/projekte/youniversity/.

44 https://www.ili.fau.de/.

45 https://www.llz.uni-halle.de/. 
- Universität Leipzig: Die LaborUniversität bietet eine hochschuldidaktische Begleitung und Vernetzung Lehrender bei der Erprobung innovativer LehrLern-Projekte sowie Transfer der Erfahrungen ${ }^{46}$.

- Universität Mainz / Hochschule Mainz: Mit der Plattform Lehrideen vernetzen soll der Transfer von guten und innovativen Lehrideen an den Mainzer Hochschulen gefördert und eine Community of Practice aufgebaut werden ${ }^{47}$ und Genius Loci-Preis für Lehrexzellenz 2018 für die Johannes GutenbergUniversität Mainz.

- Universität Utrecht: Das Teaching and Learning Lab ist eine Experimentierfläche für Lehrende, Studierende und Forschende ${ }^{48}$.

\section{Digitale Strukturen}

\section{Digitale Perspektive in Entwicklungs- und Strukturplänen präzisieren}

Zentrale Handlungsfelder der Hochschullandschaft werden heute durch digitale Entwicklungen bereichert. Es existiert eine Reihe technischer Plattformen, es wurden Kompetenzen aufgebaut, Prozesse wurden „digitalisiert“ und in einigen Hochschulen wurden Digitalisierungsstrategien entwickelt. Die Digitalisierung unterstützt nicht nur, sondern sie ermöglicht auch effizientere, komplett neue Abläufe und damit eine verbesserte Organisation des Studiums und eine Optimierung von Verwaltungsprozessen etc. Dieser Befund legt nahe, die strategischen Ziele, insbesondere auch aus der digitalen Perspektive, jeweils hochschulweit in den Blick zu nehmen. Die digitale Perspektive dieser strategischen Ziele sollte präzise, konkret und mit geeigneten Rahmenbedingungen, wie Unterfütterung mit Ressourcen und Maßnahmen, in den relevanten Papieren wie Leitbildern, Digitalisierungsstrategien oder in Entwicklungs- und Strukturplänen dargestellt werden. Aus einer eng mit den Zielen und der Profilbildung der Hochschule verbundenen Digitalisierungsstrategie lassen sich weitere Digitalkonzepte, die konkretere Ziele detaillierter beschreiben, ableiten. Die Handlungsbereiche innerhalb der Hochschule können ihre Bereichsziele, ihre eigene digitale Perspektive sowie die konkreten Maßnahmen an diesen Strategien ausrichten und einen Beitrag leisten, eine kohärenten Digitalstruktur zu entwickeln.

46 https://www.stil.uni-leipzig.de/teilprojekte/laboruniversitat/.

$47 \mathrm{https}$ ://lehrideen-vernetzen.de/.

$48 \mathrm{https} / / /$ www.uu.nl/en/education/centre-for-academic-teaching/contact/about-the-centre/ partners/teaching-learning-lab. 


\section{Profilbildung und Kooperationen entwickeln}

Die Hochschulen befinden sich bereits in einem Prozess, Strategien zu entwickeln, mit digitalen Angeboten in der Lehre zu experimentieren oder Lernprozesse digital zu unterstützen und auch zu erforschen (HFD 2016). Die durch diese Digitalisierungsprozesse einhergehenden Veränderungen innerhalb der Hochschulen bringen veränderte Rollen und Anforderungsprofile mit sich (HFD 2015). Die Digitalisierung bietet auf der einen Seite Potentiale und Möglichkeiten zur Profilierung von Hochschulen, andererseits ist der Veränderungsprozess eine große und zum Teil unübersichtliche Herausforderung.

Hochschulinterne Kooperationen bei Digitalisierungsprojekten oder Digitalisierungsprozessen befördern die Profilbildung und schaffen Transparenz. Auch die Zusammenarbeit in Kooperationen mit anderen Hochschulen bietet Möglichkeiten der Profilbildung durch Arbeitsteilung und unterstützt den Veränderungsprozess beispielsweise durch Erfahrungsaustausch und gemeinsame Konzeptentwicklungen. Diese Form der Kooperation eröffnet eine weitere Möglichkeit, gemeinsam digitale Strukturen effektiv und effizient aufzubauen. Dennoch gilt es, die Herausforderungen der digitalen Transformation zu bewältigen. Anreizsysteme für profilbildende Kooperationen oder die Anrechnung digital angereicherter Lehre können eine positive Veränderungskultur schaffen.

\section{Veränderungsprozesse mit Blick auf Digitalisierung gestalten}

Veränderungsprozesse sollten als Chance und Herausforderung betrachtet werden. Strategie- und Veränderungsprozesse sind insbesondere im Lehr-Lernkontext anzustoßen. Ziel ist die Ausschöpfung von Potentialen digitaler Bildung. Dazu bedarf es entsprechender strategischer Entscheidungen, wie beispielsweise die Anrechnung digitaler Lehre auf das Lehrdeputat. Veränderungsprozesse setzen aber auch bei der Reflektion eines neuen Rollenverständnisses von Lehrenden und Lernenden an und ermöglichen eine neue Debatte über die Kompetenzorientierung und -förderung. Ziel sollte es sein, Bottom-up- und Top-down Prozesse zu verzahnen (Liebscher et al. 2015). Dabei ist eine Verschränkung der Digitalisierungsstrategie mit veränderten Strategien in anderen Handlungsfeldern (Lehre, Verwaltung, Infrastruktur etc.) von zentraler Bedeutung. 


\section{Verbesserung der Schnittstellen gewährleisten}

Der digitale Wandel hat insbesondere an Hochschulen auf die Kernbereiche Lehre und Forschung sowie Administration starke Auswirkungen. Hochschulen sollten die Gestaltung und Entwicklung von Schnittstellen der relevanten Systeme, wie beispielsweise Learning-Management-Systeme und Campus-Management-Systeme in den Blick nehmen. Schon heute sind neben diesen klassischen Plattformen viele weitere Werkzeuge im Einsatz (Votingsysteme, Medienplattformen, Hörsaaltechnik etc.), die bereits in einer sinnvollen Umgebung eingebunden wurden. Diese Entwicklung wird sich fortsetzen und lässt sich technisch nur über Schnittstellen sinnvoll und nachhaltig abbilden. Hochschulen im 21. Jahrhundert benötigen eine Durchdringung digitaler Strukturen auf allen Ebenen - Studium, Forschung, Lehre und Verwaltung. Damit einher geht der Abbau struktureller Barrieren. Als zentrale Handlungsperspektive für die Verbesserung der Hochschulverwaltung und für die Umsetzung von Service- und Dienstleitungskonzepten dürfte sich in den nächsten Jahren insbesondere die Integration digitaler Technologien in der Lehre ergeben.

\section{IT-Infrastruktur als zentrale Stellschraube in den Blick nehmen}

IT-Infrastruktur und damit Informationstechnologie ist für Hochschulen ein unverzichtbarer Bestandteil nahezu aller Arbeitsabläufe. Der überwiegende Anteil der IT-Basisinfrastruktur wird durch zentrale Einrichtungen an Hochschulen erbracht und je nach Ausprägung der Zentralisierung der IT-Systeme stellen diese Einrichtungen fast die gesamte IT-Infrastruktur an den Hochschulen bereit. Der Betrieb heutiger und insbesondere zukünftiger IT-Systeme stellt die damit befassten zentralen Einrichtungen vor stetig wachsende Anforderungen bezogen auf Qualität, Sicherheit, Nutzbarkeit, Support und Leistungsfähigkeit moderner IT-Systeme (Linkens 2019; Goltsche 2006, 35). Diese wachsenden Anforderungen und die Nachfrage weiterer IT-Services sind geprägt von zunehmend immer kürzeren Innovationszyklen in der IT.

Die zentralen Einrichtungen sind daher in ihren Modernisierungsprozessen zu unterstützen. Veränderungs- und Modernisierungsprozesse müssen aus der Service-Perspektive gedacht und als Projekte - mit entsprechenden Ressourcen ausgestattet - umgesetzt werden. Solche Projekte hängen von vielen Parametern sowie Akteurinnen und Akteuren ab und machen eine breite Zusammenarbeit und Kommunikation erforderlich. Die Rolle des Projektmanagements sollte gestärkt werden, ihr kommt eine wesentliche Bedeutung für die Durchführung und die spätere Überführung in nachhaltige Services zu. Zukunftssichere IT- 
Einrichtungen können neben der Serviceorganisation auf eine funktionierende Projektorganisation zurückgreifen, die grundlegende Projektmanagementprozesse für Kosten, Zeitpläne, Funktionalitäten und Risiken sicher und wiederkehrend auf Projekte anwenden kann (Goltsche 2006, 18). Hochschulinterne Kooperationen, beispielsweise von Bibliotheken, Rechenzentren, Medienzentren, Didaktikzentren und Verwaltungseinheiten, können Bausteine einer zukunftsweisenden IT-Infrastruktur sein: durch die gegenseitige Unterstützung in Modernisierungsprojekten, im Qualitätsmanagement von Projekten, durch die abgestimmte Arbeitsteilung im Bereich der Services und bei gemeinsamen breiter aufgestellten Services für Lehre und Forschung.

Strategische Abstimmungsprozesse mit den Fakultäten sind ein wesentlicher Faktor, um effektiv Ziele durch solche Modernisierungen und Kooperationen zu erreichen. Neben der strategischen Ausrichtung der Ziele sollten generell die Themen Strategie, Governance, Portfolio und Budget für die IT-Infrastruktur zusammenhängend betrachtet werden.

\section{IT-Strukturen zwischen Zentralisierung und Dezentralisierung austarieren}

IT-Strategie und IT-Governance sind, neben transparenter Kommunikation zwischen den beteiligten Akteurinnen und Akteuren sowie Bereichen, hilfreiche Werkzeuge, um IT-Strukturen effektiv zu organisieren und auszutarieren. Die Aufteilung der Services und Aufgaben innerhalb des hochschulweiten IT-Portfolios und die Umsetzung von IT-Projekten haben oft einen sehr hochschulspezifischen Charakter. Die Arbeits- und Kooperationskultur dieser jeweils hochschulweiten Verteilung der Verortung entsprechender Aufgaben und Verantwortlichkeiten sollte durch strategische Steuerung und geeignete Governance-Modelle organisiert werden. Eine derart abgestimmte und strukturierte Vorgehensweise stellt langfristig sicher, dass die notwendigen Kompetenzen und die institutionellen Identitäten der Akteurinnen und Akteure möglichst optimal mit den Anforderungen der Lehrenden und Lernenden verzahnt sind und das strategische Profil der Hochschule stärken (HFD 2016, 106). Die Zusammenstellung und Entwicklung des IT-Portfolios sollte fester Bestandteil strategischer Planungen sein. Die Entwicklung und Förderung einer effektiven lokalen Kommunikationsund Organisationsstruktur kann solche Prozesse unterstützen. Die Etablierung eines Chief Information Officer (CIO), der die Abstimmung zwischen den verschiedenen Akteurinnen und Akteuren und Bereichen innerhalb der Hochschule organisiert und die strategische IT-Portfolio-Ausrichtung verantwortet, könnte ein entsprechendes Konzept sein. 


\section{Campus-Management-Systeme als zentrale Instrumente integrieren}

Die digitale Organisation des Studiums durch Prozesse entlang des sogenannten Student-Life-Cycle wird heute durch Campus-Management-Systeme in unterschiedlichen Reifegraden digital abgebildet. Diese Systeme und die damit verbundenen etablierten Prozesse sind vielerorts bereits länger im Einsatz und stellen mit der zunehmenden Digitalisierung und den zunehmenden Anforderungen an die Organisation und die Informationstechnologie die beteiligten Akteurinnen und Akteure vor große Herausforderungen. Auf der anderen Seite bietet die Modernisierung solcher Systeme der Organisationsunterstützung großes Potential zur Verbesserung von hochschulweiten Abläufen. Die Systeme werden als Kooperation der Akteurinnen und Akteure aus Fakultäten, Verwaltung und IT-Einrichtungen betrieben, damit sind Veränderungsprozesse sowohl vertikal in Richtung von Innovationen durch neue Funktionen (z. B. Schnittstellen zu weiteren IT-Systemen wie Learning-Management-Systemen und Mobilitätsanforderungen der Nutzenden) und Prozesse wie auch horizontal in Richtung von Kommunikation und Integration aller Fakultäten und deren Anforderungen an die digitale Organisation des Studiums möglich.

Diese Veränderungsprozesse können nur erfolgreich bearbeitet werden, wenn diese durch zeitaufwändige und umfangreiche Projekte, mit erheblichen Anforderungen an das Projektmanagement, an die Kommunikation mit allen relevanten Beteiligten und an die effektive Umsetzung der neuen (digitalen) Prozesse begleitet werden. Dabei kann es hilfreich sein, die Bereitstellung von Ressourcen (Personal, Zeit, Schulungen etc.) für Projektmanagement und ITProjektmanagement als langfristige Aufgabe zu erkennen. Da solche Veränderungsprojekte stark mit der Linienorganisation verbunden sind, wird eine fortlaufende Modernisierung der Einrichtungen zu einer wiederkehrenden Anforderung werden.

\section{E-Learning in den Fokus rücken}

An Hochschulen hat schon längst eine flächendeckende Erprobung und Weiterentwicklung von modernen Lehr- und Lernformaten eingesetzt. Es muss jedoch eine Klärung finanzieller und personeller Kapazitäten auf Hochschulleitungsebene diskutiert werden, um eine nachhaltige Auseinandersetzung bei der Gestaltung innovativer Lehr-Lernszenarien unter Einbezug digitaler Medien zu unterstützen. „Gute Lehre“ sollte sichtbar gemacht werden, indem sie durch Öffentlichkeitsmaßnahmen beworben und in nachhaltigen Strukturen angeboten wird. Für Hochschulen stellt sich darüber hinaus die Herausforderung, zukünf- 
tig noch stärker die Bedarfe der Studierenden bezogen auf die E-Learning-Infrastruktur in den Blick zu nehmen.

\section{Good Practice $^{49}$}

- Bundesministerium für Bildung und Forschung (BMBF): Unterstützt mit dem Programm „Digitale Hochschullehre“ die Entwicklung digitaler Lehrund Lernformate sowie die hochschulinterne Infrastruktur ${ }^{50}$.

- Deutsche Initiative für Netzwerkinformation e. V. (DINI): Setzt sich mit den Informationsinfrastrukturen an den deutschen Hochschulen auseinander, unter anderem auch bezogen auf die Gestaltung von Lernräumen ${ }^{51}$.

- Fachhochschule Bielefeld: Hat das Programm „Digitalisierung“ im Hochschulentwicklungsplan verankert ${ }^{52}$ und im Rahmen von MIND (Medienund Informationsdienste) den Serviceverbund der zentralen Einrichtungen Datenverarbeitungszentrale und Hochschulbibliothek integriert ${ }^{53}$.

- Hamburg Open Online University: Versteht sich als „Netzwerk“54 von fünf der sechs staatlichen Hamburger Hochschulen und ist an Forschungsprojekten wie BRIDGING ${ }^{55}$ beteiligt.

- HIS-Institut für Hochschulentwicklung (HIS-HE): Beschäftigt sich mit Hochschulbau und Hochschulinfrastruktur und stellt Studien zur Verfügung ${ }^{56}$.

- Hochschule Ruhr West: Das Dezernat „Innovative und nachhaltige Medien-, IT- und Informationsdienstleistungen“ ist eine zentrale Serviceeinrichtung für alle Mitglieder der Hochschule ${ }^{57}$.

49 Die Hinweise im Bereich Good Practice sind Ergebnisse der Recherchen im Rahmen des Projektes Lernwelt Hochschule. Dabei konnten sicher nicht alle Good Practice-Beispiele berücksichtigt werden. Es geht hier um Hinweise, die sicher noch ergänzt werden können.

50 https://www.bmbf.de/de/digitale-hochschullehre-2417.html.

51 https://dini.de/.

52 https://www.fh-bielefeld.de/hochschule/organisation/hochschulverwaltung/dezernat-qm/ programm-digitalisierung.

53 https://www.fh-bielefeld.de/mind.

54 www.hoou.de. Die folgende Darstellung basiert auf den Informationen, die auf der Webseite der HOOU zur Verfügung gestellt werden.

55 https://bridging.rz.tuhh.de/.

$56 \mathrm{https} / / /$ his-he.de.

57 https://www.hochschule-ruhr-west.de/die-hrw/servicebereich/dezernat-iii/. 
- Hochschulforum Digitalisierung (HFD): Stellt Studien und Arbeitspapiere für die Hochschulpraxis zur Verfügung ${ }^{58}$ und integriert die studentische Perspektive mit den \#DigitalChangemaker ${ }^{59}$.

- Leibniz-Institut für Wissensmedien: Die Digital Learning Map zeigt LehrLern-Szenarien, die digitale Medien verwenden und an deutschen Hochschulen eingesetzt werden ${ }^{60}$.

- openMINTlabs: Die Hochschulen Kaiserslautern, Koblenz und Trier entwickeln virtuelle Labore zur Vor- und Nachbereitung von Laborpraktika, indem sie Laborversuche in den Disziplinen Physik, Chemie, Biologie und den Ingenieurwissenschaften um zeitgemäße Elemente der digitalen Lehre ergänzen ${ }^{61}$.

- Technische Hochschule Wildau: Das Digital Competence Center setzt die digitale Agenda der Hochschule als strategisches Instrument zur Steigerung des Digitalisierungsgrades um $^{62}$.

- Universität Bochum: Unterstützt unter anderem mit Projekten wie 5*5000 innovative und lernförderliche eLearning-Projekte ${ }^{63}$.

- Universität Duisburg-Essen: Hat eine Strategie zur Digitalisierung in Studium und Lehre entwickelt ${ }^{64}$.

- Universität Paderborn: Versteht sich als Universität der Informationsgesellschaft ${ }^{65}$.

- Universität Trier: Hat die Koordinationsstelle E-Learning institutionalisiert ${ }^{66}$, mit vielfältigen Aktivitäten: eLITE (eLearning Infrastructure and Teaching Environment) ${ }^{67}$ und im Kontext von Studium und Lehre Projekte angestoBen $^{68}$ (Höfler-Hoang et al. 2020).

58 https://hochschulforumdigitalisierung.de/de.

59 https://hochschulforumdigitalisierung.de/de/themen/digitale-changemaker-studentischezukunfts-ag-zu-hochschulbildung-im-digitalen-zeitalter.

$60 \mathrm{https}$ //www.e-teaching.org/community/digital-learning-map/.

61 https://www.openmintlabs.de/\#top.

62 https://www.th-wildau.de/hochschule/zentrale-einrichtungen/digital-competence-center/.

$63 \mathrm{https}: / /$ www.rubel.rub.de/5x5000.

$64 \mathrm{https}: / /$ www.uni-due.de/e-learning/digitalisierungsstrategie.php.

65 https://www.uni-paderborn.de/universitaet/.

66 https://www.uni-trier.de/index.php?id=33362.

67 https://www.uni-trier.de/index.php?id=56778.

68 https://www.uni-trier.de/index.php?id=68290. 


\section{Physische Lehr- und Lernräume}

\section{Raumgestaltung als strategischen Fokus erkennen}

Hochschulen sollten in ihrer Hochschulstrategie auch eine Lernraumentwicklung berücksichtigen, um den Anforderungen durch die stetig steigenden Studierendenzahlen und den Anforderungen aus den neuen hochschuldidaktischen Veränderungen und Methoden Rechnung zu tragen. Insbesondere vor dem Hintergrund der Digitalisierung ist diese Überlegung ganzheitlich in den Blick zu nehmen (Günther et al. 2019). Insbesondere der physische Lernraum gewinnt - trotz Digitalisierung - an Bedeutung. Diesem Bedarf auf Seiten der Studierenden sollte auch unter der Perspektive der Studierendenorientierung bei allen strategischen Überlegungen Rechnung getragen werden.

\section{Lehr- und Lernumgebungen an Lernbedürfnissen und Lernprozessen orientieren}

Die Lehr- und Lernumgebungen auf die Bedürfnisse der Lernenden auszurichten, ist im Hinblick auf digitale und physische Lehr- und Lernräume von zentraler Bedeutung. Nach wie vor müssen die Studierenden - trotz der zunehmenden Digitalisierung - mit ihren analogen Bedürfnissen ernst genommen werden (Stang 2017). Dabei spielen insbesondere auch die Aufenthaltsqualitäten eine große Rolle, denn atmosphärische Qualitäten befriedigen die sozialen und emotionalen Bedürfnisse der Lernenden (Gläser/Kobsch 2020). Besonders die Veränderung von didaktischen Konzepten in Richtung Projekt- und Problembasierung erfordern für die dabei notwendigen unterschiedlichen Lernanforderungen (Gruppenarbeit, kurzfristige Recherche etc.) besondere physische Raumsettings. Deshalb sollten vielfältige Lehr- und Lernraumangebote zur Verfügung gestellt werden, um Kooperation, Kreativität und Identifikation im Studium zu befördern - einseitige Lösungen wären daher kontraproduktiv.

\section{Lehr- und Lernräume differenzieren, zonieren und flexibilisieren}

Die Differenzierung, Zonierung und Flexibilisierung von Lernräumen ist an den meisten Hochschulen bereits ansatzweise etabliert, beispielsweise in Form von Einzel- und Gruppenarbeitsplätzen oder Gruppenräumen mit Präsentationsmöglichkeiten (Aschinger 2020). Im nächsten Schritt sind innovative und explo- 
rative Konzeptelemente zu erproben. Dazu zählen beispielsweise flexible Lernräume, Makerspaces oder Learning Labs. Der innovative Ansatz muss ständig neu durchdacht werden und an die sich verändernden Bedürfnisse der Nutzerinnen und Nutzer angepasst werden. Dies erfordert neue Raum- und Möblierungskonzepte, die sich den Erkenntnissen der Lernforschung und an didaktischen Erfordernissen orientieren.

\section{Zugang zu Lehr- und Lernräumen verbessern}

Die Verbesserung der Zugänglichkeit von Lehr- und Lernräumen sollte auf zwei Ebenen optimiert werden. Zum einen sollten 24/7-Lernräume bereitgestellt werden, die mit dem Studierendenausweis zugänglich sind. Zum anderen sind Hochschulen aufgefordert, neben dem Ausbau von studentischen Arbeitsplätzen und einer vielfältigen Ausstattung auch entsprechende Informationssysteme und Lernraumübersichten zu erstellen, um eine bessere Sichtbarkeit zu erzeugen. Raumbuchungssysteme, die die Reservierung von Einzel- oder Gruppenarbeitsräumen ermöglichen, steigern Transparenz und Komfort und müssen zum Standard werden. So können sich Studierende bereits online vergewissern, ob und wo es freie Arbeitsplätze gibt.

\section{Hybride Raumstrukturen entwickeln}

Da das Zusammenspiel von digitalen und physischen Lehr- und Lernräumen bislang nur wenig entwickelt wurde, ist die Entwicklung von hybriden Raumkonzepten als eine Zukunftsaufgabe zu verstehen. Dabei sollen nicht nur elektronische Dienstleistungen in den physischen Raum integriert werden, sondern auch im virtuellen Raum auf den physischen Raum verwiesen werden. Es geht um Interaktionskonzepte an der Schnittstelle von digitaler und physischer Lernwelt. In Experimentierräumen könnten Schnittstellen identifiziert und für neue Lehr- und Lernkonzepte nutzbar gemacht werden.

\section{Hochschulweite Entwicklung von Raumkonzepten implementieren}

Auf Leitungsebene sollte eine hochschulweite Konzeptentwicklung zu physischen und digitalen Lehr- und Lernräumen mehr Berücksichtigung finden. Die strategische Bedeutung von Lehr- und Lernraumkonzepten für den Studienerfolg wird oftmals unterschätzt, die Verbindung von Lehre zum selbstbestimmten Ler- 
nen nicht offensiv gestaltet (Aschinger 2020). Insbesondere die qualitative Weiterentwicklung von Lernangeboten und Lernräumen sollte stärker in den Fokus genommen werden. Einzelinitiativen oder bilaterale Kooperationen (z. B. Rechenzentrum und Bibliothek) sollten stärker unterstützt werden. Die Koordinierung ist eine multidimensionale komplexe Aufgabe, die verschiedene an der Lernraumentwicklung beteiligte Akteurinnen und Akteure mit einbeziehen sollte.

\section{Good Practice 69}

- Deutsche Initiative für Netzwerkinformation e. V. (DINI): Die Arbeitsgruppe Lernräume beschäftigt sich mit allen Fragen der Planung und Gestaltung von Lernräumen ${ }^{70}$.

- Hochschule der Medien Stuttgart: Lernwelten werden gestaltet und erforscht (Prill 2019, 10-13).

- Hochschule München: Das transdisziplinäre Projekt „Lehrraum der Zukunft“ befasst sich mit der Wirkung des physischen Raums auf die Qualität der Lehre und den Lernerfolg der Studierenden ${ }^{71}$.

- KIT-Karlsruhe: Mit dem Projekt Learning Libraries Karlsruhe - kreativ arbeiten und lernen sollen die Karlsruher Bibliotheken als überinstitutionelle Lernlandschaft gestärkt werden ${ }^{72}$.

- SLUB Dresden: Der Makerspace ist ein offener Kreativraum für Menschen, die ihre Ideen und Do-It-Yourself-Projekte realisieren möchten ${ }^{73}$.

- SRH Heidelberg: Projekt Lernraum Campus gibt einen Überblick über Lernräume. ${ }^{74}$

- Technische Universität Eindhoven: Innovation Spaces generieren neue Formen der Zusammenarbeit zwischen Lehrenden, Studierenden und Mitarbeitenden ${ }^{75}$.

- Technische Universität München: Richtete für ihre Studierenden auf jedem Campus ein eigenes Gebäude ein. Die „StudiTUM“-Häuser sollen Platz für

69 Die Hinweise im Bereich Good Practice sind Ergebnisse der Recherchen im Rahmen des Projektes Lernwelt Hochschule. Dabei konnten sicher nicht alle Good Practice-Beispiele berücksichtigt werden. Es geht hier um Hinweise, die sicher noch ergänzt werden können.

70 https://dini.de/ag/lernraeume/.

71 https://www.hm.edu/allgemein/lehren/lehrraum_der_zukunft/index.de.html.

72 https://www.bibliothek.kit.edu/cms/laufende-projekte.php\#Anker1.

$73 \mathrm{https} / / / \mathrm{www}$.slub-dresden.de/service/arbeitsplaetze-arbeitsraeume/makerspace/.

74 https://www.hochschule-heidelberg.de/de/core-prinzip/lernraum-campus/.

75 https://www.tue.nl/en/tue-campus/tue-innovation-space/. 
fakultätsübergreifende Projekte, für spontanen Austausch und für kulturelle Aktivitäten bieten ${ }^{76}$.

- Universität Bamberg: Es werden 24/7-Lernräume angeboten ${ }^{77}$.

- Universität Basel: In der Arbeitsgruppe Lernräume arbeiten verschiedene Abteilungen zusammen an der Verbesserung der Raumsituation ${ }^{78}$.

- Universität Bielefeld: Angebote von Lernorten werden präsentiert ${ }^{79}$.

- Universität Göttingen Der Digital Creative Space ist als offen zugänglicher Co-Workingspace konzipiert ${ }^{80}$.

- Universität Hannover: Schafft eine Lernraumkarte und die Möglichkeiten der Lernraumbuchung für die gesamte Universität ${ }^{81}$.

- Universität Kassel: Mit dem LEO wird ein multifunktionaler Lernraum zur Verfügung gestellt ${ }^{82}$.

- Universität Lüneburg: Angebote von Lernorten werden präsentiert ${ }^{83}$.

\section{Fazit und Ausblick}

Ein wichtiger Aspekt im Projekt Lernwelt Hochschule war die Entwicklung eines Konzeptes für die nachhaltige Verankerung der Forschungsergebnisse sowie die Erarbeitung von Vorschlägen für ihre Umsetzung in Form von Empfehlungen und Handreichungen. Die Forschungsergebnisse wurden in einen Baukasten überführt. Vier tragende Handlungsfelder wurden identifiziert: Hochschulorganisation, Hochschuldidaktik, digitale Strukturen und physische Lehr- und Lernräume. Auf einer Metaebene wurde auch die Hochschulpolitik betrachtet und sich daraus ableitende hochschulstrategische Maßnahmen in den Blick genommen. Der hier ausgearbeitete Baukasten ist für Hochschulleitungen und an der Lernwelt Hochschule interessierte Hochschulakteurinnen und -akteure als Anregung gedacht. Die Hinweise müssen an die jeweiligen Rahmenbedingungen angepasst werden. Es geht also weniger um ein Rezeptbuch, sondern um

76 https://www.sv.tum.de/themen-projekte/studitum-haeuser-der-studierenden/.

77 https://www.uni-bamberg.de/teilbibliothek4/.

78 https://www.unibas.ch/de/Universitaet/Administration-Services/Vizerektorat-Lehre/Learning-and-Teaching/Bildungstechnologien/Lernraeume.html.

79 https://www.uni-bielefeld.de/studium/studierende/start-ins-studium/lernorte/.

$80 \mathrm{https} / / /$ www.uni-goettingen.de/de/digital+creative+space/603458.html.

81 https://www.zqs.uni-hannover.de/de/qs/lernraum/.

82 https://www.uni-kassel.de/einrichtungen/servicecenter-lehre/angebote-fuer-studierende/ leo-lernort.html.

83 https://www.leuphana.de/services/miz/lernen-arbeiten.html. 
ein Anregungsportfolio, das die Erfahrungen und wissenschaftlichen Ableitungen aus dem Forschungsprojekt Lernwelt Hochschule für eigene Vorhaben im Lernweltkontext Hochschule nutzbar macht.

Darüber hinaus wurde ein Atlas über die 2018/19 erfassten Lernwelten an deutschen Hochschulen auf der Basis der Forschungsarbeit des Projektes Lernwelt Hochschule aufgebaut und mit Daten aus weiterführenden Recherchen ergänzt. Die verschiedenen Good Practice-Beispiele stellen eine Informationsbasis dar, die idealerweise in den nächsten Jahren noch erweitert wird.

Als Provider für die dazu notwendige Internet-Plattform dient die Deutsche Initiative für Netzwerkinformation e. V. (DINI) ${ }^{84}$. Die Informationen sind auf Wiki-Basis aufbereitet ${ }^{85}$.

$\mathrm{Zu}$ einem Nachhaltigkeitskonzept gehört neben dem technischen auch ein organisatorischer Rahmen. Im Projekt Lernwelt Hochschule sind die dafür notwendigen Prozesse erarbeitet und beschrieben worden. Sie werden ab 2020 von der DINI-Arbeitsgruppe Lernräume fortgeführt.

\section{Literatur}

Aschinger, F. (2020): Konzeption und Management der Lernwelt Hochschule. Herausforderungen und Good Practice aus Sicht der Hochschulakteurinnen und -akteure. In: A. Becker; R. Stang (Hrsg.): Lernwelt Hochschule. Dimensionen eines Bildungsbereichs im Umbruch. Berlin; Boston: De Gruyter Saur, 123-149.

Babyesiza, A.; Berthold, C. (2018): Tatsächliche Hochschulautonomie am Beispiel der finanziellen Steuerung der Hochschulen in Brandenburg und Nordrhein-Westfalen. Arbeitspapier Nr. 206. Güterloh: CHE. https://www.che.de/downloads/CHE_AP_206_Finanzautonomie. pdf.

Barr, R. B.; Tagg, J. (1995): From Teaching to Learning. A New Paradigm for Undergraduate Education. Change: The Magazine of Higher Learning 27/6, 12-26, DOI: 10.1080/ 00091383.1995.10544672.

Becker, A.; Stang, R. (Hrsg.) (2020a): Lernwelt Hochschule. Dimensionen eines Bildungsbereichs im Umbruch. Berlin; Boston: De Gruyter Saur

Becker, A.; Stang, R. (2020b): Lernwelt Hochschule im Aufbruch. Zentrale Ergebnisse einer Befragung. In: A. Becker; R. Stang (Hrsg.): Lernwelt Hochschule. Dimensionen eines Bildungsbereichs im Umbruch. Berlin; Boston: De Gruyter Saur, 71-122.

Brahm T.; Jenert T.; Euler D. (2016): Pädagogische Hochschulentwicklung als Motor für die Qualitätsentwicklung von Studium und Lehre. In: Brahm T.; Jenert T.; Euler D. (Hrsg.): Pädagogische Hochschulentwicklung. Wiesbaden: Springer, 19-36.

84 Deutsche Initiative für Netzwerkinformation e. V. (DINI). http://www.dini.de. 85 https://leho-dini.de. 
Burmester, M.; Seidl; T. (2020): Lehr-Lernkontexte in einer transformativen Fakultät. Konzeptionelle Perspektiven. In: R. Stang; A. Becker (Hrsg.): Zukunft Lernwelt Hochschule. Perspektiven und Optionen für eine Neuausrichtung. Berlin; Boston: De Gruyter Saur, 86-95.

Gläser, C.; Kobsch, L. (2020): Student Experience in der Lernwelt Hochschule. Studierende im Fokus der Fallstudien. In: A. Becker; R. Stang (Hrsg.): Lernwelt Hochschule. Dimensionen eines Bildungsbereichs im Umbruch. Berlin; Boston: De Gruyter Saur, 150-169.

Goltsche, W. (2006): COBIT kompakt und verständlich. Wiesbaden: Springer Fachmedien.

Günther, D.; Kirschbaum, M.; Kruse, R.; Ladwig, T.; Prill, A.; Stang, R.; Wertz, I. (2019): Zukunftsfähige Lernraumgestaltung im digitalen Zeitalter. Thesen und Empfehlungen der Ad-hoc Arbeitsgruppe Lernarchitekturen des Hochschulforum Digitalisierung. Arbeitspapier Nr. 44. Berlin: Hochschulforum Digitalisierung. https://hochschulforumdigitalisierung.de/sites/default/files/dateien/HFD_AP_44-Zukunftsfaehige_Lernraumgestaltung_Web.pdf.

HFD - Hochschulforum Digitalisierung (2015): Diskussionspapier. 20 Thesen zur Digitalisierung der Hochschulbildung. Arbeitspapier Nr. 14. Berlin: Hochschulforum Digitalisierung. https://hochschulforumdigitalisierung.de/sites/default/files/dateien/HFD\%20AP\%20 Nr \%2014_Diskussionspapier.pdf.

HFD - Hochschulforum Digitalisierung (2016): The Digital Turn. Hochschulbildung im digitalen Zeitalter. Arbeitspapier Nr. 27. Berlin: Hochschulforum Digitalisierung. https://hochschulforumdigitalisierung.de/sites/default/files/dateien/Abschlussbericht.pdf.

Höfler-Hoang, B.; Röder; D.; Ertz, F. (2020): Digitalisierung als Teil der Universitätsentwicklung. Strukturen, Angebote und Ziele der Universität Trier. In: R. Stang; A. Becker (Hrsg.): Zukunft Lernwelt Hochschule. Perspektiven und Optionen für eine Neuausrichtung. Berlin; Boston: De Gruyter Saur, 35-43.

Kuhn, M.; Nitsche-Ruhland, D.; Klein, J. (2020): Neue Lernwelten etablieren. Forschungsintegrierte Lehre an der DHBW. In: R. Stang; A. Becker (Hrsg.): Zukunft Lernwelt Hochschule. Perspektiven und Optionen für eine Neuausrichtung. Berlin; Boston: De Gruyter Saur, 7785.

Liebscher, J.; Petschenka, A.; Gollan, H.; Heinrich, S.; van Ackeren, I.; Ganseuer, C. (2015): ELearning-Strategie an der Universität Duisburg-Essen. Mehr als ein Artefakt? Zeitschrift für Hochschulentwicklung (ZFHE) 10/2, 96-109.

Linkens, H.-J. (2019): Qualitätsanforderungen für Data Science in Deutschland. In: Gesellschaft für Informatik (Hrsg.) (2019): Data Literacy und Data Science Education. Digitale Kompetenzen in der Hochschulausbildung. Berlin: Gesellschaft für Informatik, 19-21. https://gi. de/fileadmin/GI/Hauptseite/Aktuelles/Aktionen/Data_Literacy/GI_DataScience_2018-0420_FINAL.pdf.

Mildenberger, U.; Vonhof, C. (2020): Neues Studienmodell und organisatorische Herausforderungen. Wege zu einer transformativen Fakultät. In: R. Stang; A. Becker (Hrsg.): Zukunft Lernwelt Hochschule. Perspektiven und Optionen für eine Neuausrichtung. Berlin; Boston: De Gruyter Saur, 26-34.

Ninnemann, K. Rózsa, J.; Sutter, C. (2020): Zur Relevanz der Verknüpfung von Lernen, Raum und Organisation. Paradigmenwechsel vom Lehren zum Lernen an der SRH Hochschule Heidelberg. In: R. Stang; A. Becker (Hrsg.): Zukunft Lernwelt Hochschule. Perspektiven und Optionen für eine Neuausrichtung. Berlin; Boston: De Gruyter Saur, 179-190.

Prill, A. (2019). Lernräume der Zukunft. Vier Praxisbeispiele zu Lernraumgestaltung im digitalen Wandel. Arbeitspapier Nr. 45. Berlin: Hochschulforum Digitalisierung. https://hoch- 
schulforumdigitalisierung.de/sites/default/files/dateien/HFD_AP_45-Lernraeume_der_Zukunft_Praxisbeispiele_Web.pdf

Radcliffe, D.; Wilson, H.; Powell, D.; Tibbetts, B. (Hrsg.) (2009): Learning Spaces in Higher Education. Positive Outcomes by Design. Proceedings of the Next Generation Learning Spaces 2008 Colloquium. Queensland, Australia: The University of Queensland.

Stang, R. (2017): Analoger Körper im digitalen Raum: Lernen im Zeichen einer ambivalenten Kontextualisierung. In: F. Thissen (Hrsg.): Lernen in virtuellen Räumen. Perspektiven des Mobilen Lernens. Berlin; Boston: De Gruyter Saur, 28-38.

Stang, R.; Becker, A. (Hrsg.) (2020): Zukunft Lernwelt Hochschule. Perspektiven und Optionen für eine Neuausrichtung. Berlin; Boston: De Gruyter Saur.

Stang, R., Becker, A. Franke, F. Gläser, C., Petschenka, A., Weckmann, H.-D., Zulauf, B. (2020): Herausforderungen Lernwelt Hochschule. Perspektiven für eine zukünftige Gestaltung. In: A. Becker; R. Stang, R. (Hrsg.): Lernwelt Hochschule. Dimensionen eines Bildungsbereiches im Umbruch. Berlin; Boston: De Gruyter Saur, 182-210.

Weichert, H. (2020): Strukturentwicklungspläne und Leitbilder. Orientierungen für strategische Planungen. In: A. Becker; R. Stang (Hrsg.): Lernwelt Hochschule. Dimensionen eines Bildungsbereichs im Umbruch. Berlin; Boston: De Gruyter Saur, 59-70.

Weichert, H.; Stang, R. (2020): Der Blick von außen. Einschätzungen internationaler Expertinnen und Experten. In: A. Becker; R. Stang (Hrsg.): Lernwelt Hochschule. Dimensionen eines Bildungsbereichs im Umbruch. Berlin; Boston: De Gruyter Saur, 170-181. 